\title{
Convolutional neural networks for global human settlements mapping from Sentinel-2 satellite imagery
}

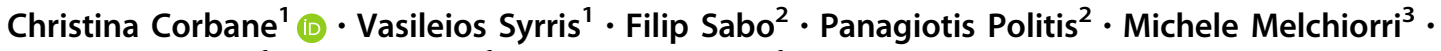 \\ Martino Pesaresi ${ }^{1} \cdot$ Pierre Soille $^{1} \cdot$ Thomas Kemper $^{1}$
}

Received: 18 May 2020 / Accepted: 14 October 2020/Published online: 27 October 2020

(C) The Author(s) 2020

\begin{abstract}
Spatially consistent and up-to-date maps of human settlements are crucial for addressing policies related to urbanization and sustainability, especially in the era of an increasingly urbanized world. The availability of open and free Sentinel-2 data of the Copernicus Earth Observation program offers a new opportunity for wall-to-wall mapping of human settlements at a global scale. This paper presents a deep-learning-based framework for a fully automated extraction of built-up areas at a spatial resolution of $10 \mathrm{~m}$ from a global composite of Sentinel-2 imagery. A multi-neuro modeling methodology building on a simple Convolution Neural Networks architecture for pixel-wise image classification of built-up areas is developed. The core features of the proposed model are the image patch of size $5 \times 5$ pixels adequate for describing built-up areas from Sentinel-2 imagery and the lightweight topology with a total number of 1,448,578 trainable parameters and 4 2D convolutional layers and 2 flattened layers. The deployment of the model on the global Sentinel-2 image composite provides the most detailed and complete map reporting about built-up areas for reference year 2018. The validation of the results with an independent reference dataset of building footprints covering 277 sites across the world establishes the reliability of the built-up layer produced by the proposed framework and the model robustness. The results of this study contribute to cutting-edge research in the field of automated built-up areas mapping from remote sensing data and establish a new reference layer for the analysis of the spatial distribution of human settlements across the rural-urban continuum.
\end{abstract}

Keywords Convolutional neural networks · Remote sensing · Image segmentation · Human settlements · Built-up areas

\section{Introduction}

New ways to map and measure the built-up environment over large areas are critical to answering a wide range of research questions and to addressing policies related to urbanization and sustainability. This is particularly true in the era of an increasingly urbanized world [1]. Earth

Electronic supplementary material The online version of this article (https://doi.org/10.1007/s00521-020-05449-7) contains supplementary material, which is available to authorized users.

Christina Corbane

Christina.corban@ec.europa.eu

1 European Commission, Joint Research Centre (JRC), Ispra, Italy

2 Arhs Developments S.A, 4370 Belvaux, Luxembourg

3 Engineering S.p.a, 00144 Rome, Italy
Observation (EO) has become a promising tool to provide up to date geospatial information on the status and dynamics of built-up areas and human settlements [2]. With the routine acquisition of satellite imagery and the availability of different satellite collections, several efforts have focused on mapping built-up areas at a global scale in the last decade. The most recent datasets include the Global Urban Footprint (GUF) with its $12 \mathrm{~m}$ product derived from TerraSAR-X imagery acquired in 2011-2013 [3]; the Global Human Settlement Layer (GHSL) with the $30 \mathrm{~m}$ multitemporal datasets derived from Landsat archives and showing the evolution of built-up areas in four epochs 1975, 1990, 2000 and 2014 [4, 5]; the World Settlement Footprint (WSF) with the $10 \mathrm{~m}$ resolution datasets based on Landsat-8 and Sentinel-1 sensors for reference year 2015 [6] and the FROM-GLC10 landcover map which includes a dedicated class for artificial surfaces derived from Sentinel2 data acquired in 2017 [7]. Unlike the GUF which was generated from commercial imagery, all the other products 
were derived from free and open-access satellite image datasets, primarily from Landsat and the European Copernicus Sentinel missions. The advantages of these products are numerous and are mainly related to their free availability (absence of restrictions on their use for multiple types of applications) and most of all for the relatively low cost of their systematic update.

The methods used to produce these products and in general to extract built-up areas or artificial surfaces from remote sensing data include statistically derived indices and both supervised and unsupervised learning approaches. The first group of methods covers typically spectral indices [8-10], spectral mixture analysis $[11,12]$ and local/contextual image contrast/texture analysis [13, 14]. The latter includes regression analysis [15-17] and machine learning techniques, comprising mostly decision trees and random forests [18-20], support vector machines [7] and associative rule learning $[4,5]$.

Although some of these methods have proved to be suitable for large-area mapping of human settlements from satellite imagery, several limitations must be considered when using the information products generated from public satellite data for analytical purposes. These limitations are mostly related to accuracy, sensor-scale dependency, mapping of the extrema of the settlement density range, and the continuous monitoring of urban land cover changes. A non-exhaustive list follows below:

- High false positive and false negative error rates from the automated detection of urban land cover classes when compared to non-urban classes (e.g., bare rocks, sand dunes, bare agricultural fields, river bank lines) due to the limited actual extent of built-up areas and the discontinuous surface they compose [21];

- High disagreement on total land cover surface estimates of different sensor-derived products and high dependency on input sensor resolution of the urban land cover total estimates [22-24];

- Unsatisfactory mapping of the extrema of the settlement spatial patterns at the very low-density rural areas and the very high-density urban areas [25-27];

- Lack of a commonly approved methodology and/or a machine-based automatic and reproducible solution which allows consistent and continuous monitoring of global urban land cover changes across time and across different sensors [2, 28, 29].

Compelling challenges and opportunities still lie ahead in high-resolution mapping and accurate classification of built-up areas over large areas. A key issue in this context is up-to-date and reliable information on the status and development of the human settlements. The availability of free and open remotely sensed big data streams has brought significant innovations in the field of automatic information extraction from satellite imagery. There is an increasing need to mine the large amount of earth observation data delivered in a free and open way by some of the new generation of satellites, especially the Sentinel missions. Operational since 2017, the Sentinel-2 mission of the European Copernicus program provides a 5-day repeat cycle and a span of 13 spectral bands at a spatial resolution as high as $10 \mathrm{~m}$. Sentinel-2 has great potential for mapping and monitoring built-up areas on a global scale [7, 30, 31]. Novel approaches for mapping human settlements are needed to deal with the increased spatial and temporal resolution of Sentinel-2.

\subsection{Background}

Advances in deep learning (DL) have led to leaps in the fields of computer vision, speech recognition and natural language processing.

Whereas the task of built-up areas extraction from remote sensing data has a number of unique challenges, primarily related to the sensor and the features to be detected, it draws concepts and theories from computer vision, signal processing, statistics and machine learning [38]. Since 2014, the remote-sensing community has shifted its attention to DL for addressing different application domains [32] such as image registration [33], image fusion [34, 35], change detection [36] and object detection [37]. However, image classification is the remote sensing field where DL has gained most of its popularity [32].

Recent applications in remote sensing have used DL approaches for image classification tasks at which the purpose was the labeling of single pixels or regions of an image according to two or more classes [39-41]. DL methods have experimentally proved to outperform stateof-the-art machine learning methods (e.g., Support Vector Machines, Random Forests) [42] for the classification of both optical (hyperspectral and multispectral imagery) [41, 43], radar imagery [44], change detection [45] and for the extraction of different land cover types such as roads [46], crop types [40] and buildings [47].

Ball et al. (2017) [38] provide a comprehensive survey of image classification works in remote sensing that rely on DL approaches, while the review paper of Ma et al. (2019) [42] on DL approaches covers nearly every application and technology in the field of remote sensing, ranging from preprocessing to image fusion, object detection and land cover mapping. A recent study suggested that deep learning is suitable for capturing the fine features of complex urban areas and performs better than conventional thresholdbased methods, traditional supervised classifications and machine learning approaches [48]. In particular, architectures building on convolutional neural networks (CNNs) have become viable solutions for remote sensing image 
classification where traditional handcrafted feature engineering and domain-knowledge methods fail because of the limited generalization capabilities of the algorithms, the inter-class similarity, the intra-class variability as well as the changing image acquisition conditions [49, 50].

Differently from other DL approaches, deep CNNs were specifically designed for image classification; nevertheless, they can be easily adapted to solve image segmentation problems by performing pixel-wise classification [51]. The hierarchical features of the input image data are modeled naturally by the CNN hierarchical structure, a fact that boosts the CNN performance in satellite image classification in general and facilitates the extraction of built-up features in particular. Another main advantage of CNN architectures over other established methods used for generating the global maps of built-up areas is their capacity to be integrated with mature frameworks of image pre-processing and standardization tools providing shiftinvariant and contrast-invariant image local transforms [52].

Recognizing the inherent advantages of convolution operations in the characterization of the built-up environment in remote sensing data, a significant amount of works have recently explored the potential of diverse CNN architectures for mapping built-up areas from different types of sensors and different spatial resolutions: Synthetic Aperture Radar [44], high and very high spatial resolution imagery [48, 53, 54] and aerial imagery [55] (i.e., with a ground sampling distance equal to or even less than $1 \mathrm{~m}$ ). However, little effort has been directed toward the challenge of large-scale built-up areas mapping with CNN from data of lower spatial resolution such as the ones powered by Sentinel-2. The works of $[56,57]$ represent a significant advancement in that direction. In particular, the framework of human settlements mapping proposed at $20 \mathrm{~m}$ by [57] is a step-forward toward a global scale model. Despite the demonstrated generalization and upscaling capabilities of their proposed framework, the authors failed to implement the CNN model in rural areas, which represent one of the main challenges in built-up areas mapping from satellite data at global scale.

\subsection{Challenges addressed in this work}

When deploying CNNs on large geographical areas or at global scale, four main issues should be taken into consideration:

- The necessity to develop a model flexible enough to be applied to a global carpet of satellite data entailing the design of a sound training approach, a strategy for transfer learning and a plan for the consistency verification of the classification output.
- The substantial amount of training data required for training complex models. In the case of built-up classification, the training samples should cover different building types (e.g., residential and industrial buildings of different sizes, colors and rotations) in various types of landscapes (e.g., dense urban areas, rural areas, desert landscapes, built-up areas mixed with neighborhood green spaces);

- The increased need for computational processing resources, especially for adjusting and fine-tuning multiple and/or complex models;

- The requirement for $\mathrm{CNN}$ architectures that are robust to noise in satellite imagery (e.g., presence of snow, clouds, haze) and to other seasonal effects. This feature would enable the generalization capacity of the models over large areas and the extraction of built-up areas with comparable efficacy along the urban-rural continuum.

In this work, we propose a Neural Computing framework tailored for global scale mapping human settlements at a spatial resolution of $10 \mathrm{~m}$, from a cloud-free composite of Sentinel-2 data for reference year 2018. The output is a global map of built-up areas expressed in terms of a probability grid.

The main contributions of the work can be summarized as follows:

- A new framework for pixel-wise large-scale classification of built-up areas from a Sentinel-2 image composite at a spatial resolution of $10 \mathrm{~m}$ has been developed, named GHS-S2Net (GHS stands for Global Human Settlements, S2 refers to the Sentinel-2 satellite) (Sect. 2.3);

- A multi-neuro modeling methodology is proposed following the Universal Transverse Mercator (UTM) grid zones schema and a systematic two-stage sampling within each UTM grid zone (Sect. 2.3.1);

- Transfer learning is implemented following two separate approaches depending on the availability of reliable training data at the different UTM zones: a close range transfer learning within each UTM grid zone and a far range transfer learning from one UTM grid zone to neighboring data-poor zones (Sect. 2.3.3). In this work, transfer learning does not obey the most dominant definition of using the weight values of pre-trained models from different domains. As a concept herein, it is closer to the verification of the generalization capacity of the models when the training and testing data do not necessarily follow similar statistical distributions;

- An extensive assessment of the models output, that is based on an independent validation using fine-scale digital cartographic reference data reporting the 


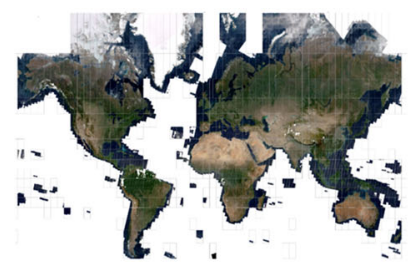

Input Sentinel-2 global composite at 10 meters

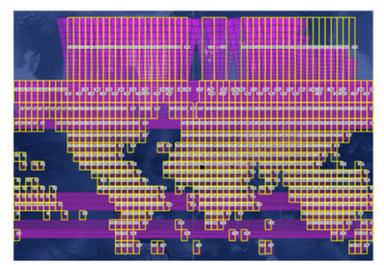

Multi-modelling schema: single model per each UTM grid zone

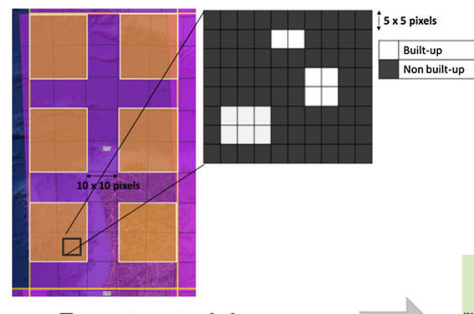

Two-stage training

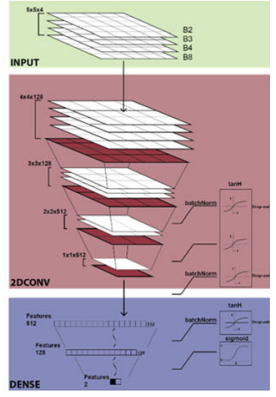

GHS-S2Net

Training of 485 models

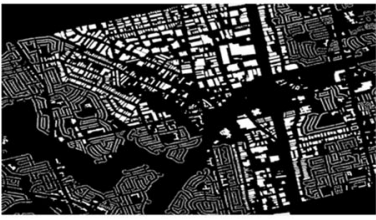

Validation of model predictions against building footprints

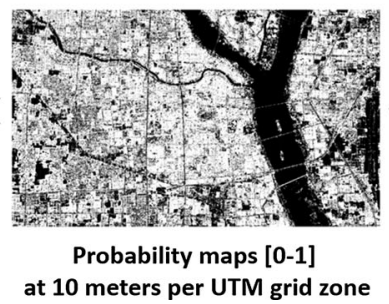

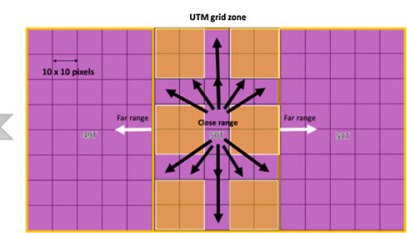

Per-tile predictions: Close range and Far range transfer learning

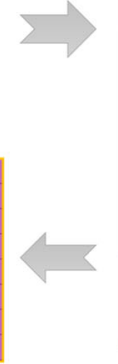

at $\mathbf{1 0}$ meters per UTM grid zone

Fig. 1 Overview of the proposed framework for global human settlements mapping from Sentinel-2 image composite

footprint of every single building for 277 sites around the globe (Sect. 3.4).

The proposed framework for built-up areas classification at global scale is summarized in the illustrative diagram of Fig. 1. Details on each of the processing steps are provided in the subsequent sections.

The new framework leverages the JRC Big Data Platform (JEODPP) [58] for the storage of the global input data and for optimized fast parallel processing using the highperformance Graphics Processing Units (GPUs). This dedicated infrastructure allows tackling the challenges of large-scale processing, boosting the CNN training, and enhancing the prediction accuracy through duly fine-tuning of the models.

\section{Input data and methods}

\subsection{Sentinel-2 cloud-free image composite}

The input data for human settlements mapping over the entirety of the landmass (excluding Antarctica) consist in a global cloud-free image composite for reference year 2018 derived from Sentinel-2 satellite data of the European Copernicus Earth observation program. Sentinel-2 mission offers a great potential for fine-scale mapping and monitoring of built-up areas thanks to high spatial and temporal resolutions, with a five-day revisit time and decametric resolution [31]. However, the selection of the best available scenes, their download from the dedicated data hubs together with the requirements in terms of storage and computing resources pose restrictions for large-scale mapping. Pixel-based compositing is an approach to leverage the large volumes of available data, while effectively mitigating cloud and aerosol contamination as well as data gaps in the archive [59]. This method has been recognized for being a valuable tool for large area applications using high spatial resolution optical data [60]. Accordingly, the image composite was generated in and exported from Google Earth Engine [61]. The methodology used for the selection of the satellite imagery and for image compositing is based on a data-driven approach which uses a summary statistic for aggregating the pixel time series (i.e., the 25th percentile). A detailed description of the workflow is presented in [62]. The output image composite consists of a global scale raster grid of four spectral bands derived from top of atmosphere Sentinel-2 image tiles (B2: Blue, B3: Green, B4: Red and B8: Visible and Near Infrared) with a spatial resolution of $10 \mathrm{~m}$. It was produced and tiled following the UTM system with each tile having the projection of the UTM zone (UTM/WGS84 projection) to which it corresponds to. There are in total 615 grid zones with data covering mostly mainland and islands (Fig. 2). The full dataset has a total volume of $15 \mathrm{~TB}$ and is hosted on the Big Data platform of the Joint Research Centre (JEODPP). The raster data have been stored in 16-bit geotiff format. The dataset can be freely accessed and downloaded from the Open Data Catalogue of the Joint Research Centre of the European Commission ${ }^{1}$ [63].

\footnotetext{
${ }_{1}$ https://data.jrc.ec.europa.eu/dataset/0bd1dfab-e311-4046-8911c54a8750df79.
} 


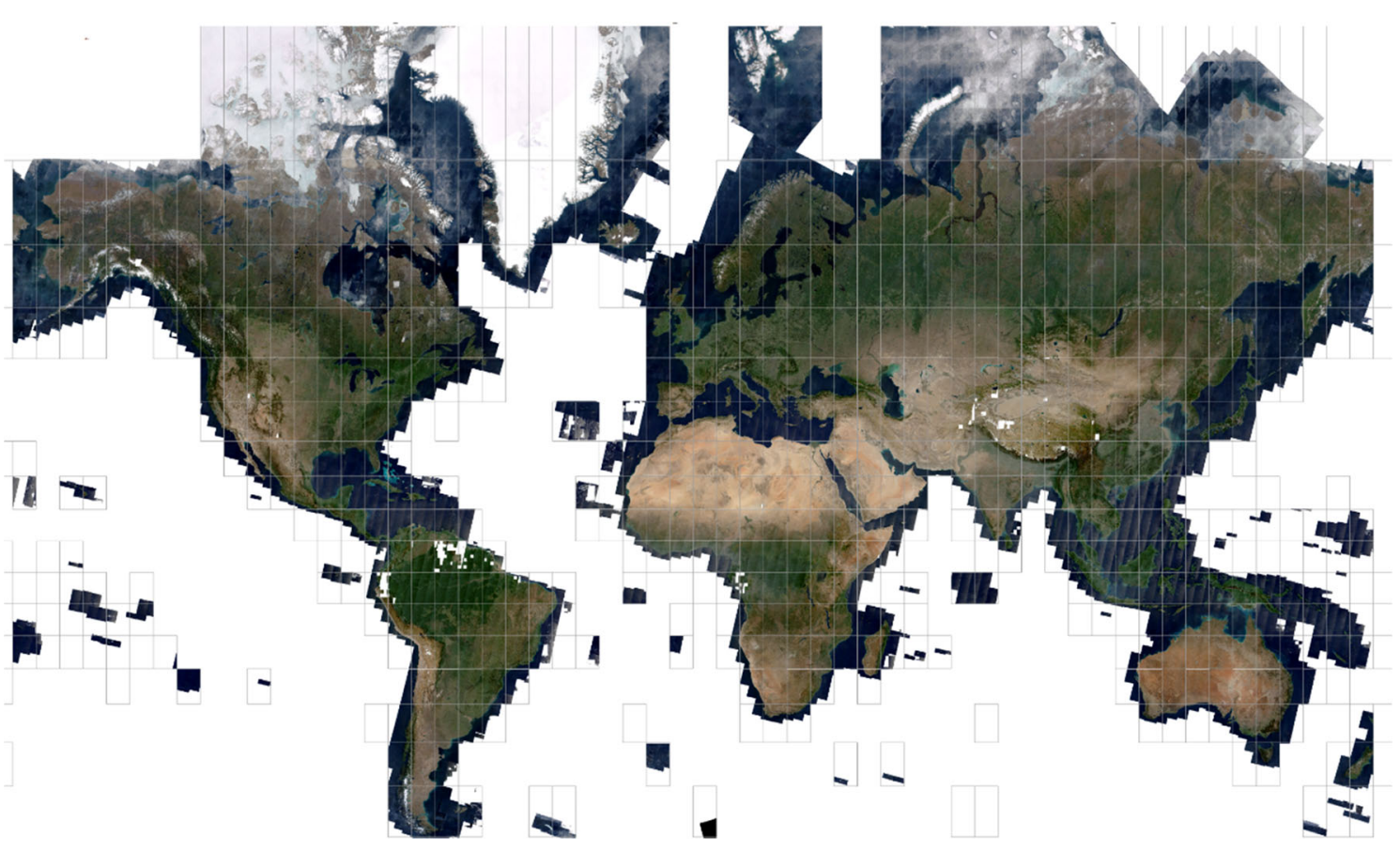

Fig. 2 Overview of the cloud-free Sentinel-2 image composite organized by UTM grid zones

\subsection{Model input data: learning sets}

A sensitive point regarding CNNs is the amount of training data required to properly adjust the network parameters. A large source of free and open access datasets describing built-up areas was collected with different levels of details, completeness, consistency and accuracy. Since the aim is to achieve a stable and at the same time detailed and accurate delineation of built-up areas, the most detailed datasets describing built-up areas were compiled from public sources: The Global Human Settlement Layer (GHSL_BU), the European Settlement Map (ESM_BU), the Facebook high resolution settlement data (FB_HRS) and the Microsoft building footprints (MS_BFP) described hereafter.

\subsubsection{Global Human Settlement Layer built-up areas}

GHSL_BU was derived from automatic classification of Landsat $30 \mathrm{~m}$-resolution data of the year 2014 as described in [5]. The method for mapping built-up areas from Landsat data at global scale builds on the Symbolic Machine Learning (SML) classifier which automatically generates inferential rules linking the image data to available high-abstraction semantic layers used as training sets [64]. The product is provided with a spatial resolution of $30 \mathrm{~m}$. Despite the overall good performance in depicting built-up areas at global scale, the GHSL_BU suffers from under-detection problems in sparsely built-up areas and mainly in rural African landscapes.

\subsubsection{European Settlement Map}

ESM_BU is the $2 \mathrm{~m}$ resolution land cover class "built-up area" produced by the automatic classification of the Copernicus VHR_IMAGE_2015 collection which covers 39 European countries (EEA39) with various earth observation sensors. The built-up areas extraction has been achieved through supervised learning with the SML classifier along with textural and morphological features [65]. The ESM_BU is currently the most detailed map of builtup areas available for Europe. The main issue in this layer is the presence of false alarms, in particular over mountainous areas and sand beaches as well as the absence of cloud-free satellite data in some regions resulting in large data gaps observed in certain urban areas (e.g., UK (Manchester, Peterborough, Reading, Luton, Coventry) and Ireland (Dungarvan)).

\subsubsection{Facebook high-resolution settlement data}

The FB_HRS data used in the study are derived from the high-resolution settlement grids produced by Facebook [66]. The dataset was made available for public use in the frame of "Data for Good" Facebook program that supports international humanitarian efforts [67]. The settlement areas of FB_HRS were automatically delineated by a $\mathrm{CNN}$ classifier working over sub-meter resolution optical satellite imagery and using fine-scale open-source training data as Open Street Map (OSM) [68]. The $30 \mathrm{~m}$ spatial resolution derived data [67] have been used in the present 
study. At the time we compiled the FB_HRS data, 150 countries were covered by the FB_HRS including large parts of South America, Africa, Europe and Asia. According to the information available on a subset of 194 countries, the image data supporting the FB_HRS spatial delineation were collected in the time range from 2002 to 2017, with a temporal surface-weighted average centered in the year 2013. Based on our internal quality control procedure, the precision of these data was particularly remarkable in rural areas flagging (at a spatial resolution of $30 \mathrm{~m}$ ) the presence of single isolated houses and small rural hamlets precisely. Commission errors were noticed occasionally in rural areas, especially in correspondence with dense forest patterns. The mapping of large urban areas as accounted by the FB_HRS data turned to be more problematic; in these areas, remarkable systematic omission errors were noticed.

\subsubsection{Microsoft building footprint data}

The MS_BFP is a vector data derived from the work of the Microsoft map team and available for public use in the OSM community. The data were automatically extracted by the Open Source CNTK Unified Toolkit developed by Microsoft. CNTK and the ResNet34 with RefineNet upsampling layers were applied to detect building footprints from the Bing imagery that may include VHR satellite and airborne sensors [69]. The MS_BFP data were made available in vector format at a nominal scale of 1:10.000, thus supporting a detailed rasterization at $1 \times 1 \mathrm{~m}$ of spatial resolution successively aggregated to $10 \times 10 \mathrm{~m}$ resolution used in this study. At the time we compiled the MS_BFP data, information about four countries was available: United States, Canada, Uganda and Tanzania. Despite the detailed representation of single buildings, the MS_BFP data suffer from omission errors referring to large industrial buildings and fewer errors related to over-detections of buildings in mountainous and agricultural areas.

Table 1 gives an overview of the specific training sets used for adjusting the models with respect to the following characteristics: spatial resolution, coverage, source image collection date used for layer production, identified issues as well as the number of pixels (total and relative percentages) used as training samples. Figure 3 displays the selected information sources for training the models by geographic area.

Due to the overall quality and spatial detail of the training data and to the variability in both the spatial coverage and the type of issues associated with each dataset, a hierarchical process was implemented for selecting the best data available locally: the priority was given first to MS_BFP and ESM_BU which are the closest proxies to the built-up areas to be derived from $10 \mathrm{~m}$ resolution satellite data. They were followed by the FB_HRS and finally by the GHSL_BU, which is the least detailed representation of built-up areas.

\subsection{GHS-S2Net building blocks}

The purpose of the proposed CNN model named here GHSS2Net is to perform pixel-wise classification of built-up areas at a spatial resolution of $10 \mathrm{~m}$. The concept of "builtup area" applied here is consistent with the definition adopted in the framework of GHSL which is "the union of all the satellite data samples that corresponds to a roofed construction above ground which is intended or used for the shelter of humans, animals, things, the production of economic goods or the delivery of services" [70].

Pixel-wise grouping is equivalent to the standard image segmentation process, i.e., partitioning of the image into multiple segments corresponding to individual pixels or homogenous areas. GHS-S2Net architecture builds on the CNN configurations described in [71]. A schematic representation of the GHS-S2Net is visualized in Fig. 4. The two major drivers that framed the design of this $\mathrm{CNN}$ model are explained below:

- Firstly, given that the target to be recognized ranges in size from single residences until block of contiguous buildings, the model capacity should allow the collection and distillation of the fine information provided by either the single pixels or the small sized groups of pixels consisting of homogeneous characteristics. Unlike popular tasks for natural image segmentation and object localization where there exist sizeable image regions with common characteristics (color, texture, connectivity, etc.), the size of the objects to be recognized herein varies from $10 \mathrm{~m}$ (the finest resolution associated with a single pixel) to some dozens of meters. Consequently, the contextual information that surrounds one pixel and accommodates the prominent features can be expressed by narrow image windows (patches) having a size of few pixels. An extensive experimentation specifically for Sentinel-2 imagery with respect to the optimal size of an image patch at which the convolution performs efficiently is presented in [71]. In the present study, an image patch of size $5 \times 5$ has been selected as input image to the CNN, whereas the convolution of the image is achieved through successive kernels of size $2 \times 2$ with stride $1 \times 1$. At this narrow representation and with the intention of avoiding losing essential information, no pooling layers have been employed to reduce further the spatial size.

- Secondly, the motivation was to design a lightweight model that could serve adequately the chosen multi- 
Table 1 Summary characteristics of the training sets

\begin{tabular}{|c|c|c|c|c|c|c|c|}
\hline \multirow[t]{2}{*}{$\begin{array}{l}\text { Training } \\
\text { set }\end{array}$} & \multirow[t]{2}{*}{ Pixel size (m) } & \multirow[t]{2}{*}{ Coverage } & \multirow[t]{2}{*}{$\begin{array}{l}\text { Time } \\
\text { stamp }\end{array}$} & \multirow[t]{2}{*}{ Advantages } & \multirow[t]{2}{*}{ Constraints } & \multicolumn{2}{|c|}{$\begin{array}{l}\text { BU samples } \\
\text { (resampled at } \\
10 \mathrm{~m})\end{array}$} \\
\hline & & & & & & $\begin{array}{l}\text { Number } \\
\text { of pixels }\end{array}$ & $\%$ \\
\hline GHSL_BU & 30 & Global & 2014 & $\begin{array}{l}\text { Complete global } \\
\text { coverage }\end{array}$ & $\begin{array}{l}\text { Lower spatial resolution than the } \\
\text { data under processing, thus } \\
\text { including relatively higher error } \\
\text { rates }\end{array}$ & $1.49 \mathrm{E}+09$ & 28.29 \\
\hline ESM_BU & 2 & European & 2015 & $\begin{array}{l}\text { High precision from } \\
\text { very higher } \\
\text { resolution input data }\end{array}$ & $\begin{array}{l}\text { Limited geographical coverage, } \\
\text { large no data zones over some } \\
\text { cities }\end{array}$ & $5.31 \mathrm{E}+08$ & 10.04 \\
\hline FB_HRS & $\sim 30$ & $\begin{array}{l}194 \\
\quad \text { countries }\end{array}$ & 2002-2017 & $\begin{array}{l}\text { High precision derived } \\
\text { by aggregation of } \\
\text { very higher } \\
\text { resolution input data }\end{array}$ & $\begin{array}{l}\text { Limited geographical availability, } \\
\text { systematic false negative in dense } \\
\text { urban areas, sporadic false } \\
\text { positives }\end{array}$ & $2.59 \mathrm{E}+09$ & 49.06 \\
\hline MS_BFP & $\begin{array}{l}\text { vector (rasterized } \\
\text { at } 1 \mathrm{~m} \text { and } \\
\text { aggregated to } 10 \\
\mathrm{~m} \text { ) }\end{array}$ & 4 countries & - & $\begin{array}{l}\text { High precision with } \\
\text { delineation of single } \\
\text { buildings from very } \\
\text { high resolution input } \\
\text { data }\end{array}$ & $\begin{array}{l}\text { Limited geographical availability, } \\
\text { sporadic false negative in } \\
\text { industrial areas, sporadic false } \\
\text { positives in specific landscapes } \\
\text { (Canadian lakes, mountainous } \\
\text { areas), unknown imagery date }\end{array}$ & $6.66 \mathrm{E}+08$ & 12.61 \\
\hline
\end{tabular}

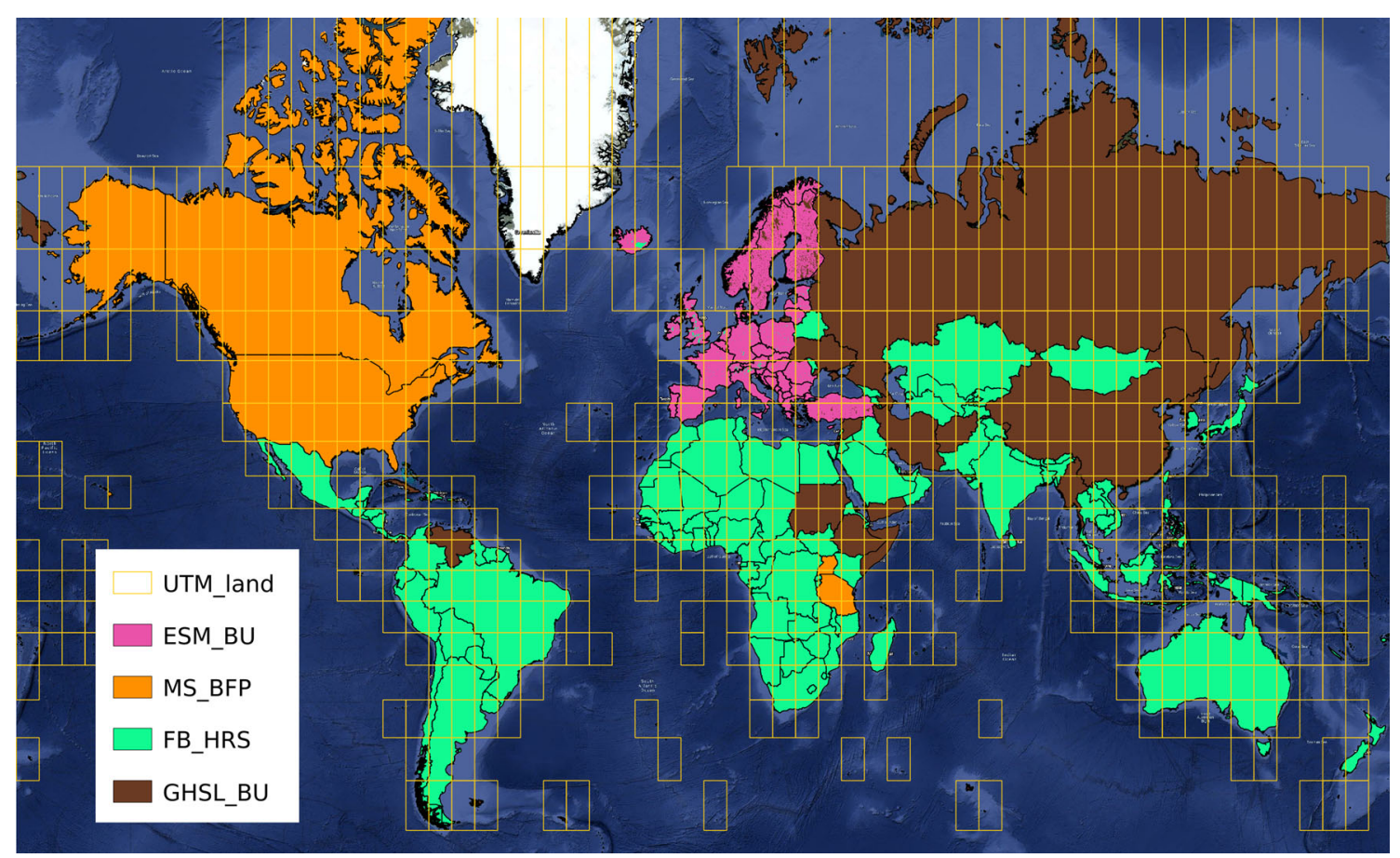

Fig. 3 Spatial distribution of the training datasets at country level. The colors correspond to the valid data masks of the learning sets over land (color figure online)

modeling approach and allow several degrees of flexibility in terms of distributed computing. The total number of model parameters is $1,448,578(1,447,042$ trainable and 1,536 non-trainable), 95 times less than VGGNet [72] and 2.7 times less than GoogleNet [73] (indicative CNNs). While the number of $2 \mathrm{D}$ convolutional layers is limited to 4 layers and the number of flattened layers to 2 , the number of parameters has been increased due to the high number of filters. Tests showed that the specific CNN topology can perform 


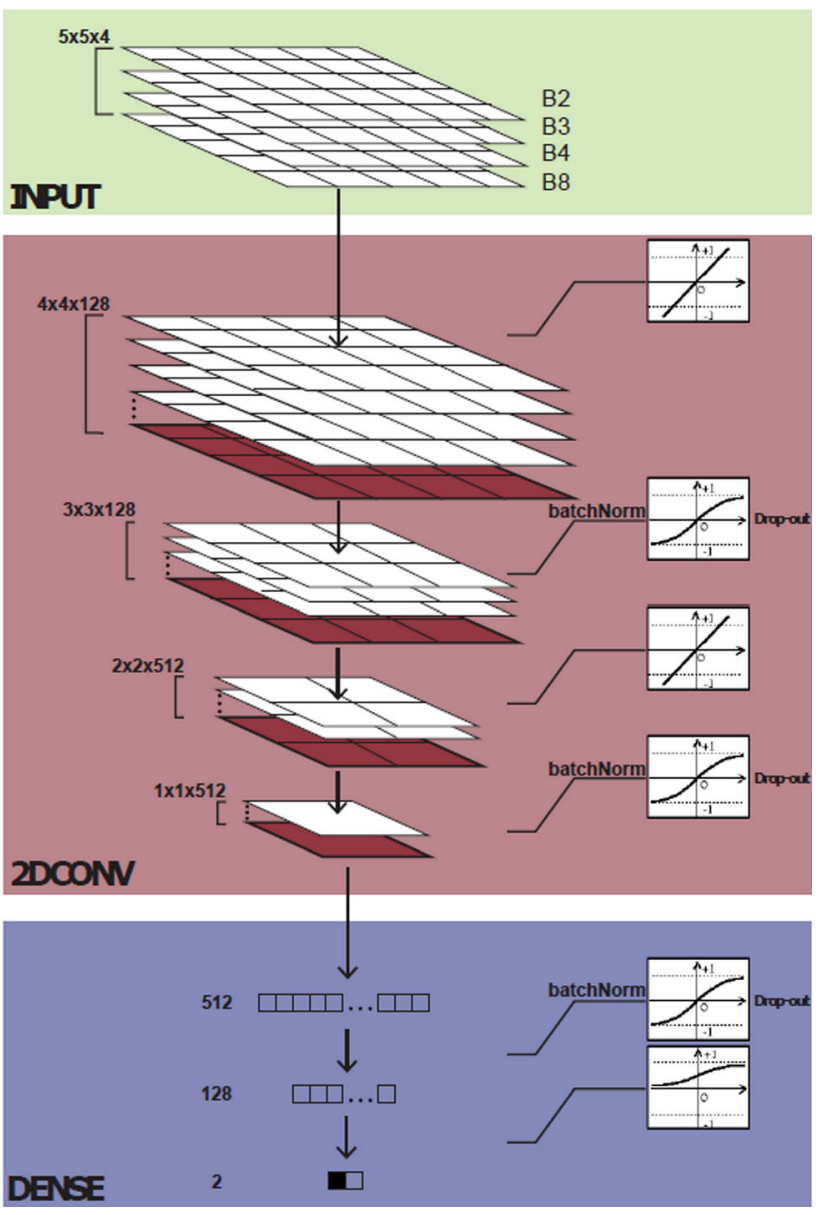

Fig. 4 Schematic representation of the layers that compose the GHSS2Net architecture

quite well even if the number of filters is smaller, yet we decided to keep the number of filters high in order for the model to capture very subtle details. This lightweight topology facilitates the algorithm execution across heterogeneous GPU modules throughout the prototyping and operational phase. Additionally, it enables smoothly the multi-modeling deployment at which a different model has been trained over every UTM zone, capturing more precisely the local characteristics and the variance along similar geographical regions.

The $2 D C O N V$ block as shown in Fig. 4 comprises two successive stacks where $2 \times 2$ convolution takes place and the linear and the hyperbolic tangent activation functions (tanh), respectively, transform the signals across the network layers. Although the rectifier activation function and its variants have been used widely in the various deep neural network architectures due to their robustness against the vanishing gradient problem [74], our experimentation indicated that by using a smaller number of neural network layers, the functional mapping via tanh activations captures better the complexity of the features with respect to the Sentinel-2 imagery. Besides, the tanh function is more suitable in the case of optimization with stochastic gradient descent where sigmoid function shows sharp damp gradients during backpropagation as well as gradient saturation [75]. The alternation with linear mappings results in a costeffective solution in terms of computations. Speed-up of the training process and remedy to the effect of the internal covariate shift is provided through data batch normalization operations [76]; at each data batch, transformation is performed by keeping mean activation close to 0 and the activation standard deviation close to 1 . A subsequent dropout regularization layer [77] has been used to prevent overfitting, with a ratio of 0.1 of neurons not considering at each update during the training phase.

The sigmoid function has been employed only for the last layer and maps the model output into the range [0,1], giving rise to the probability of a pixel to belong to the class built-up.

\subsubsection{Two-stage training approach}

We propose a two-stage training approach at which a single model per each UTM grid zone has been trained in accordance with the zones used for generating the Sentinel2 image composite. This multi-modeling approach aims at capturing the variations in the Sentinel-2 data and the diverse characteristics of human settlements (in terms of size, shape, morphology and structure). Furthermore, rather than training a very complex single model that would need big volumes of representative data, the training of several relatively light CNNs facilitates the modeling of local features and distributes effectively the computational load into several machines by increasing significantly the total throughput. Each UTM grid zone covers an average area of $447,650 \mathrm{~km}^{2}$ (area calculated in equal area projection). This type of data splitting is prone to containing various types of built-up areas and settlement patterns across heterogeneous landscapes even within the same UTM zone. Besides, the semantic classes of "built-up" and "non built-up" are unevenly distributed spatially and their frequencies are highly varying. The class "built-up" is very rare compared to the non-built-up class (See Supplementary material R1) $(2 \%$ of the training samples $(5 \times 5$ pixel blocks) represent built-up, while $98 \%$ represent non-builtup). To tackle this uneven distribution of training samples, each UTM grid zone was split into tiles of $100 \times 100 \mathrm{~km}^{2}$, which is consistent with the tile size of the Sentinel-2 granules (purple cells in Fig. 5). The two stages are described below: 


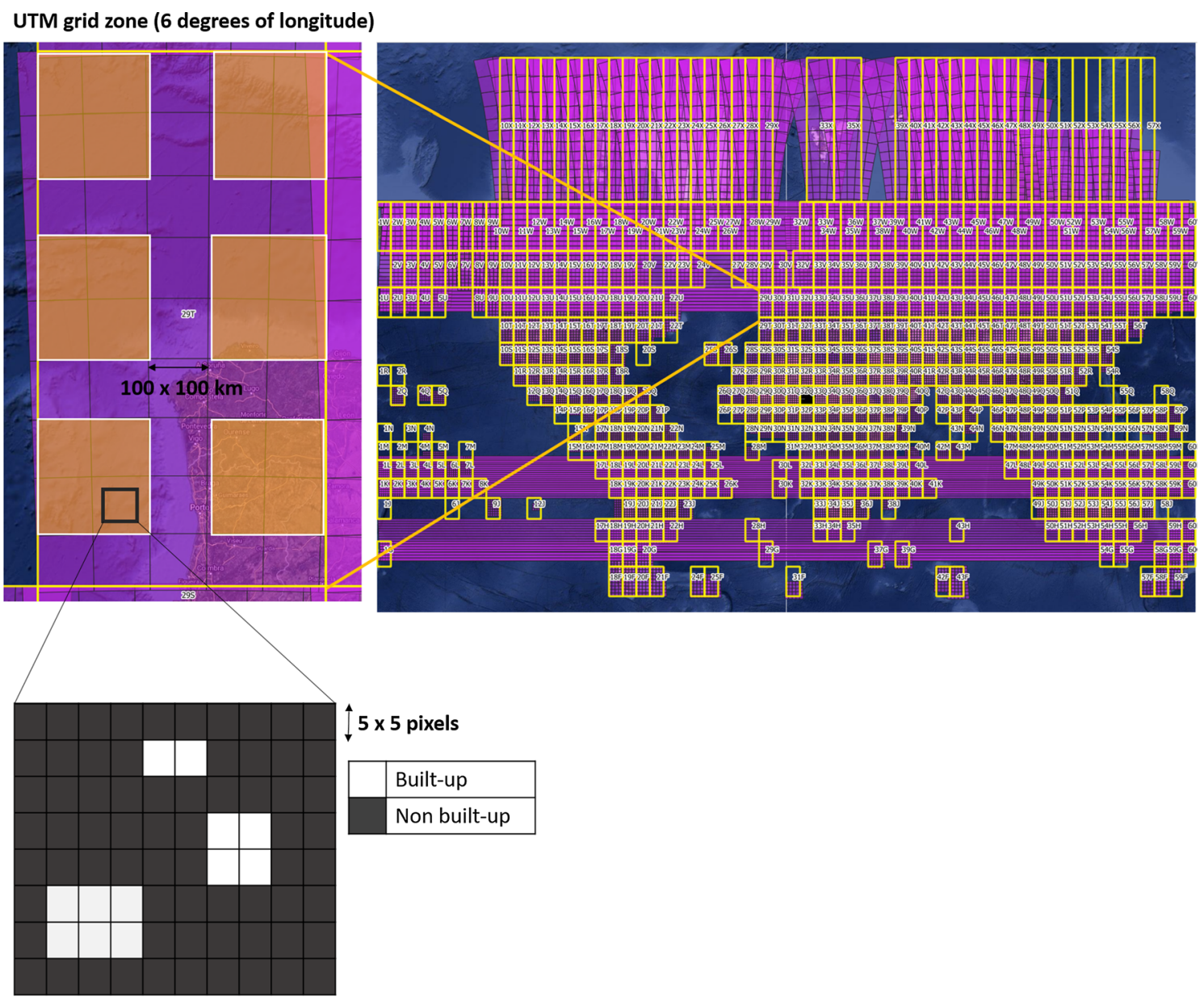

Fig. 5 Two-stage training in which one model is trained per UTM grid zone (color figure online)

(1) select systematically $50 \%$ of the $\sim 100 \times 100 \mathrm{~km}^{2}$ tiles of the UTM grid zones for the model training (orange boxes in Fig. 5);

(2) consider all built-up patches $(5 \times 5$ blocks of pixels of $10 \mathrm{~m}$ containing at least one built-up pixel) falling within the selected $100 \times 100 \mathrm{~km}^{2}$ tiles and randomly sample $60 \%$ of the non-built-up patches uniformly with respect to their frequency in the tile (checkerboard in Fig. 5). The training of the models per UTM grid zones is done by grouping the built-up and non-built-up patches into mini-batches of 200,000 samples (where the steps per epoch depend on the training size of each UTM zone) as a compromise between computational constraints and the need to converge to a global optimum. A special attention is given to UTM grid zones largely covered by water surfaces and no data in the Sentinel-2 image composite. In such cases, all the tiles of the valid data domain are considered in the training phase without applying any sampling approach.

\subsubsection{Per-tile predictions}

As described previously, the CNN model consists of encoding layers solely, through which the information existing into image blocks of size 5 rows $\times 5$ columns $\times 4$ bands is multiplexed and transformed to a single value, denoting the probability of the central pixel of the $5 \times 5$ block to belong to the built-up class. The prediction phase has been performed with tiles of size 10,000 rows $\times$ 10,000 columns $\times 4$ bands. A sliding window of size $5 \times 5$ pixels has been applied to produce the 5 rows $\times 5$ columns $\times 4$ bands input blocks. Constant-value image padding has been also implemented in order for the pixels at the image border to be correctly inserted into the $5 \times 5 \times 4$ input blocks. The predictions of the model are given in vector format having exactly the same size as the rows and columns product of the original input tile.

\subsubsection{Close range and far range transfer learning}

Transfer learning is a paradigm in DL to solve a target problem by reusing the learning with minor modifications 
from a different but related source problem. Qin et al. [78] review transfer learning in remote sensing applications and categorize the methods into four families depending on what is being transferred:

- instance-based transfer which uses partial training samples in the source domain to improve the performance of the model of the target domain [79];

- feature representation-based transfer [80] which assists the target domain classifier to learn a more effective feature expression from the source domain and improve its performance;

- relational knowledge transfer [81] where knowledge among the data in the source domain is transferred to the target domain;

- parameter-based transfer [82] considers that the source domain classifier and target domain classifier have the same optimal parameters, which can be found from the source domain classifier and then used for the target domain classifier.

Another more general classification of transfer learning methods considers the availability of labeled data and categorizes the methods into three sub-settings [83]: inductive transfer learning, when labeled data in the target domain are available; transductive transfer learning, when solely labeled data in the source domain are available; and unsupervised transfer learning, when labeled data do not exist in either the source or target domain.

One of the goals of this work is to address the following aspects of the pixel-wise classification: the computation time for training a big number of models for every UTM grid zone and the availability and precision of the training data. Parameter-based transfer learning was adopted in a transductive transfer learning framework tailored to the training strategy described in the Sect. 2.3.1. This includes a close range and a far range transductive transfer of model parameters (Fig. 6):

- The close range transfer learning consists in training the model with a subset of the input data in a given UTM grid zone (following the method described in Sect. 2.3.1) and applying it to all the $100 \times 100 \mathrm{~km}^{2}$ tiles falling within the same UTM grid zone. This approach allows speeding up the training process of 485 different models and producing the predictions of a total of 30,000 tiles. It also helps overcoming overfitting issues;

- The far range transfer learning consists in training the model with detailed samples such as MS_BFP and FB_HRS in a given UTM grid zone and applying it to a neighboring zone or to zones with similar landscape and built-up typology, at which labeled samples are scarce or zones where only GHSL_BU training datasets are available. This approach allows refining the predictions and testing the generalization capabilities of the $G H S$ S2Net model.

\subsection{Processing infrastructure}

The computing-intensive workflow was executed on the JEODPP infrastructure. The JEODPP is a versatile platform with multi-petabyte scale storage (14 PiB currently) co-located with computational capabilities [58]. The platform is based on commodity hardware and open-source software stack including the EOS storage technology developed by the European Organization for Nuclear Research (CERN) [84]. The platform has been recently upgraded with a series of GPU nodes to speed-up machine/ deep learning applications. Currently, there are 5 GPU nodes equipped with different types of GPU modules and
Fig. 6 Example of close range and far range transfer learning according to the two-stage training approach. Close range transfer learning is performed in this illustrative example within UTM grid zone $50 \mathrm{~T}$ and far range transfer learning is done by transferring the model parameters from UTM grid zone $50 \mathrm{~T}$ to nearby zones $49 \mathrm{~T}$ and $51 \mathrm{~T}$

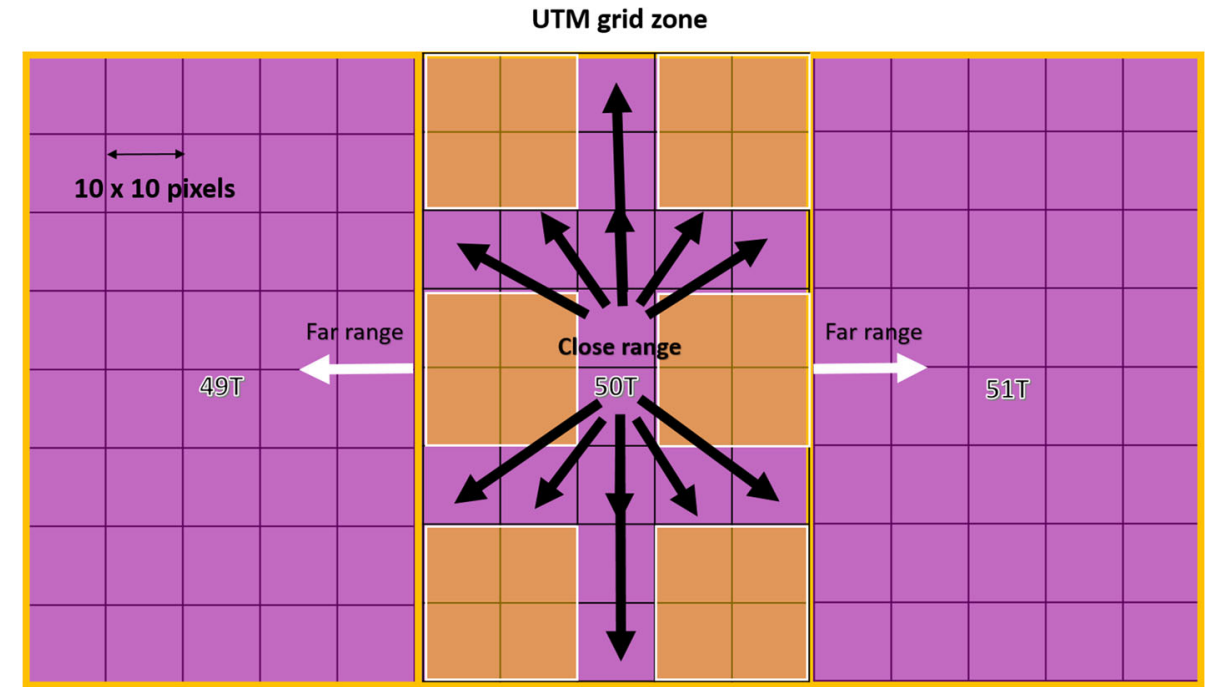


memory per module. For the training of the GHS-S2Net models, as well as for the prediction phase, 2 GPU nodes were used: the first with 4 Quadro RTX 6000 with 24.2 GB of memory and the second with $2 \mathrm{~T}$ V100-PCIE with 32.5 GB of memory. Dedicated Docker images integrating the necessary deep learning packages were created to run all the experiments.

\section{Results}

\subsection{Training phase of CNN models per UTM grid zone}

\subsubsection{Hyper-parameters tuning}

During the training phase of the model per each UTM grid zone, $10 \%$ of the training data were reserved for validation in order for the CNNs to prevent over-fitting. The input Sentinel-2 composite data were rescaled in the range $[0,1]$. The number of epochs to train the models was set to 25 iterations. The weights were initialized based on uniform distribution with bounds $[-0.1065,0.1065]$. Finally, the Adam stochastic optimization with a learning rate of 0.0001 has been used to optimize the binary cross-entropy, $\log$ loss function:

$L(\mathrm{y}, \hat{y})=-\frac{1}{N} \sum_{n=1}^{N}\left[y_{n} \log \hat{y}_{n}+\left(1-y_{n}\right)\left(1-\log \hat{y}_{n}\right)\right]$

where $N$ is the number of training samples, $y$ is the vector of the real target values of the training set in binary coding, and $\hat{y}$ is the vector of the model responses in the continuous range $[0,1]$. The cross-entropy loss has fast convergence rate and is numerically stable when coupled with sigmoid normalization [85].

\subsubsection{Performance evaluation}

For evaluating the classification performance of the models during the training and prevent overfitting, a fraction representing $10 \%$ of the training data was used for validation. Figure 7 shows the progress of the average loss curves produced by 485 GHS-S2Net models during their training and validation which last 25 epochs. Every model corresponds to one UTM grid zone, resulting in 485 out of 615 grid zones that refer to landmass with presence of built-up according to the learning sets. The learning curves show that both the average training loss (green curve) and validation loss (red curve) decrease rapidly to a point of stability with a convergence around 12 epochs. The fact that the gap between the two curves is very small even for the first 5 iterations and that it completely disappears around
12 iterations after, shows that the size of the training sets, selected following the two-stage training approach, is optimal and that the models have good generalization capacity [86].

\subsection{Computational performance of the GHS- S2Net models during the training and prediction phases}

Both training and prediction were performed on GPUs and their runtime is reported in Fig. 8. The reported elapsed time refers to every UTM grid zone predominantly covered by land (204 grid zones) and those zones predominantly covered by water ( 281 grid zones). In inland tiles, more training samples are usually fed to the GHS-S2Net, while in water tiles the number of training samples is smaller. The stacked bar plots show that the average training time is around $3600 \mathrm{~s}$, while the prediction time is around $15,500 \mathrm{~s}$. For inland zones, the average training time is $3900 \mathrm{~s}$ and the prediction time is $16,400 \mathrm{~s}$, while for water zones, the processing time is shorter with an average training time of $3100 \mathrm{~s}$ and prediction time of $15,000 \mathrm{~s}$.

These results show that the GHS-S2Net-based multimodeling approach scales seamlessly in a distributed multiGPU platform. For the processing at a global scale, our main constraint was the limited amount of concurrently available GPUs: we employed 6 GPU modules for the training phase and 2 modules for the prediction phase that were available at the time of deployment. Despite these limitations, we managed to scale up the GHS-S2Net-based multi-modeling approach and achieved to process a dataset having global coverage at $10 \mathrm{~m}$ spatial resolution thanks to: (i) an efficient partitioning of the processing per UTM grid zone, (2) the two-stage training approach with a subsampling of non-built-up patches within the selected tiles containing training samples, and (3) the optimal size of input data (i.e., $100 \times 100 \mathrm{~km}$ tiles) used for both the training and prediction. Increased GPU capacities and activation of early stopping during the training in order to reduce the number of iterations (epochs) when the loss function stops improving, can significantly reduce both the training and the prediction time of the GHS-S2Net model.

\subsection{Qualitative assessment of the models predictions}

The results of the GHS-S2Net implementation on the Sentinel-2 global mosaic were assessed visually. Compared to the training sets, the results of built-up detection showed a significant reduction in both commission and omission errors and other artifacts that were observed in the training sets (see Sect. 2.2). In addition, GHS-S2Net resulted in a refined mapping of built-up areas and open spaces within 


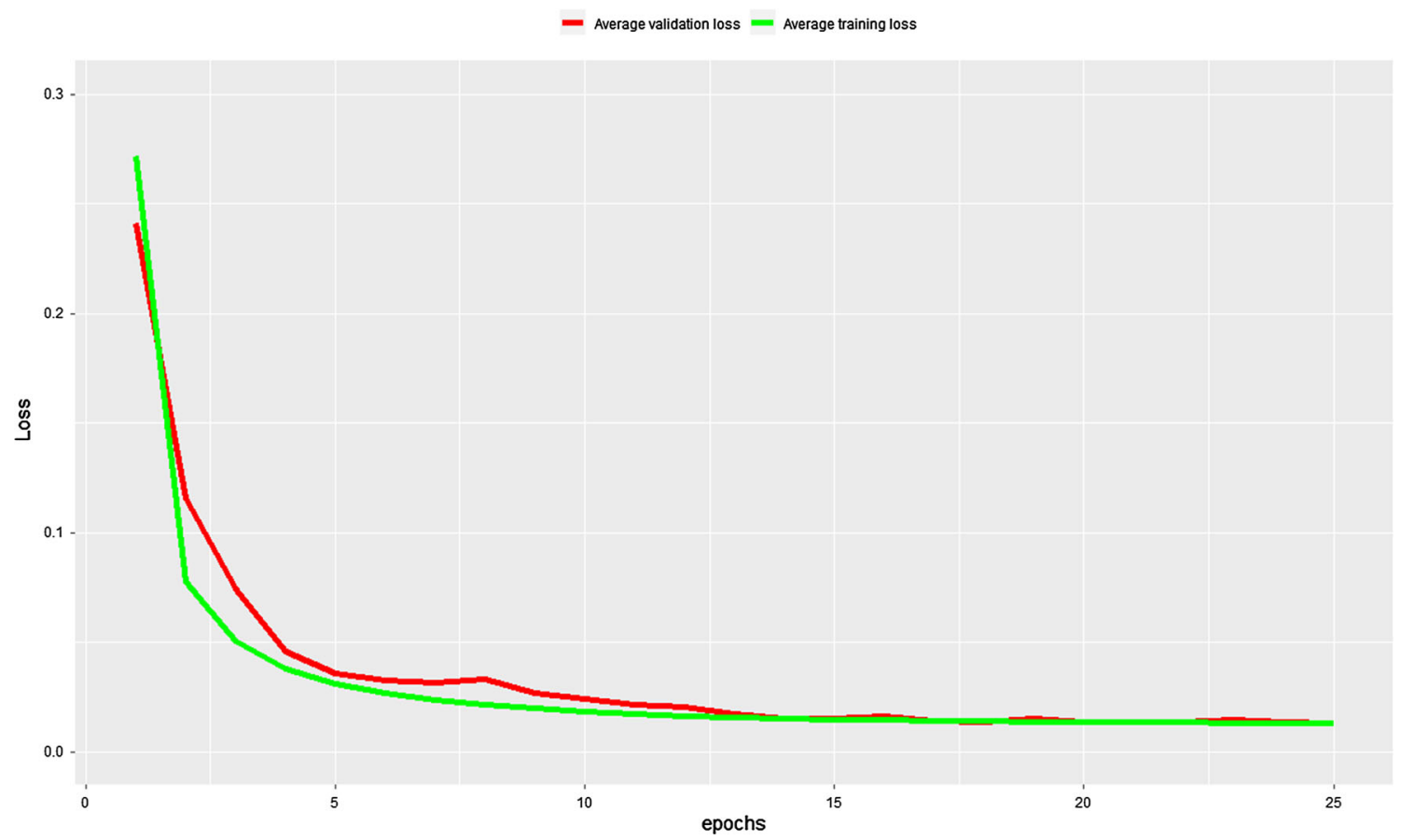

Fig. 7 Average loss curves produced by 485 GHS-S2Net models during their training and validation, where each model corresponds to a different UTM grid zone (color figure online)

urban areas and most importantly the detection of new settlements, never annotated so far in the training sets or identified in any other global scale dataset. Figure 9 illustrates some examples of each type of improvement obtained with the GHS-S2Net models. Figures 9, 10 and 11 show, for selected cities, the enhanced built-up areas detection, represented in the form of continuous-range outputs (probability), in comparison with the best available training sets. The most notable improvements relate to the detection of built-up areas which are omitted from the training sets, under the assumption that the initial purpose of these datasets was to map completely the contiguous areas they cover. These omissions are either due to lack of data or to flaws and gaps in the training sets themselves given that they were all extracted through automatic classification of satellite imagery. In the case of FB_HRS (Fig. 9a: 7.34 Latitude, 3.90 Longitude), the most critical omissions were systematically observed in dense built-up areas (often corresponding to urban cores), while in ESM_BU (Fig. 9b: 51.44 Latitude, -0.97 Longitude), the omissions were essentially due to lack of input satellite data in some countries (mainly United Kingdom and Ireland). In the case of MS_BFP (Fig. 9c: 43.11 Latitude, -79.05 Longitude), most of the omissions concerned large industrial buildings but several small buildings were also not detected in this training data. For GHSL_BU (Fig. 9d: 30.51 Latitude, 120.67 Longitude), underdetections were mainly observed in rural areas and in particular in small scattered settlements due to the size of the built-up structures which were difficult to be captured due to the sensor's spatial resolution.

Figure 10 is another example highlighting the capacity of the GHS-S2Net in reducing significantly commission problems observed in the training sets that were fed to the models. In the case of MS_BFP, overdetections were mainly observed in mountainous areas with bare rocks or in agricultural areas with bare fields (Fig. 10a: 33.25 Latitude, -90.62 Longitude). In the case of ESM_BU, overdetections were frequently identified in sand dunes (Fig. 10b: Latitude 43.36, 16.65 Longitude) and rocky beaches, bright bare soils and riverbeds.

The visual comparison of the results of the GHS-S2Net probabilistic output against the best available training sets provides a clear evidence of the refined built-up areas detection from the Sentinel-2 image composite. Figure 11 is an example of such enhanced capabilities covering the city of Sassari (Italy). It compares the ESM_BU training set derived from VHR satellite data at a spatial resolution of $2 \mathrm{~m}$ to the results obtained by the GHS-S2Net trained with ESM_BU. These results illustrate the unprecedented performance of GHS-S2Net for pixel-wise classification of $10 \mathrm{~m}$ Sentinel-2 data and for detecting urban structures in complex urban environments. Not only the classification of built-up areas is more refined, despite the coarser spatial resolution of Sentinel-2 data $(10 \mathrm{~m})$ in comparison with the VHR imagery used for producing ESM_BU (2 m) 
Processing time per UTM grid zone inland

Training_time Prediction_time

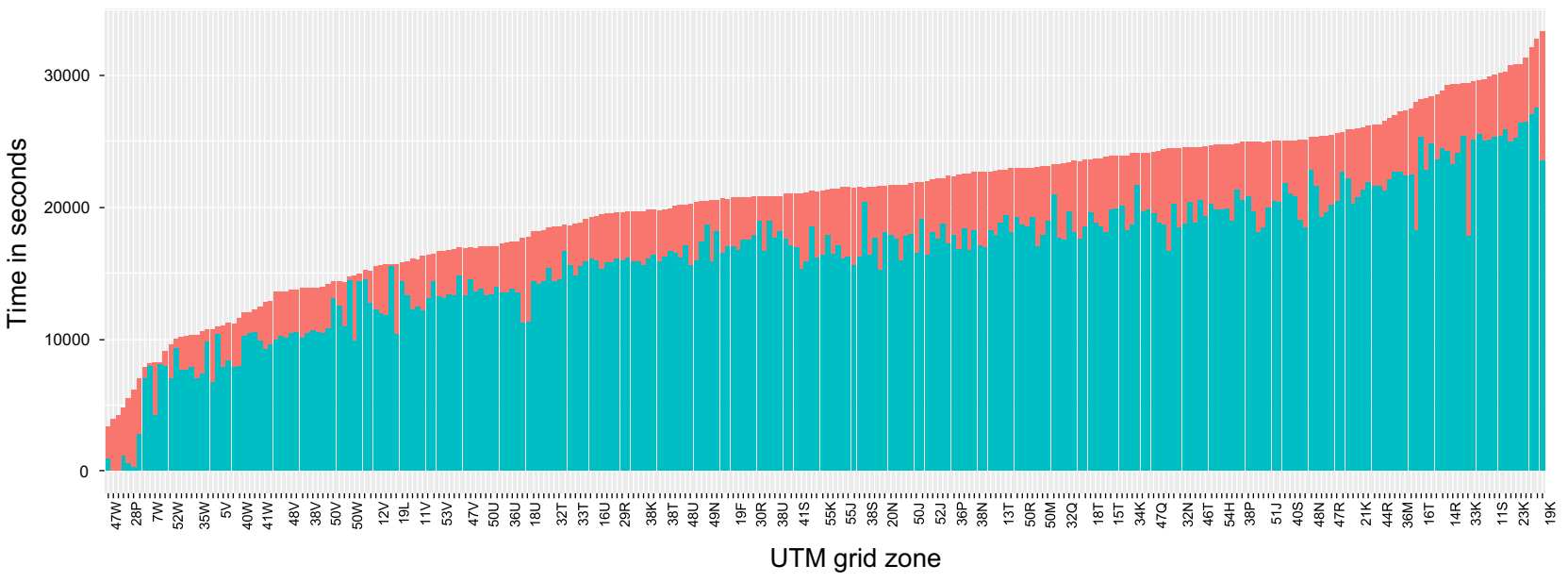

Processing time per UTM grid zone water

Training_time Prediction_time

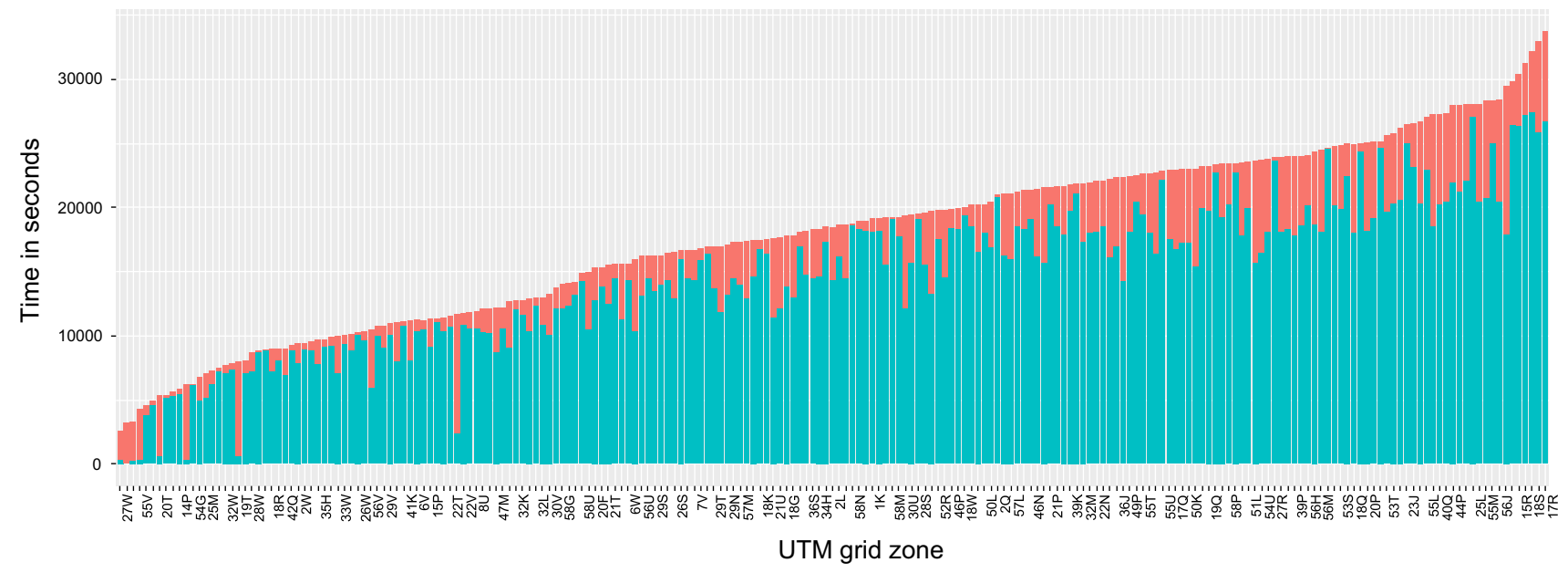

Fig. 8 Training and prediction runtime per UTM grid zone. The upper figure refers to inland dominated grid zones and the bottom figure to the water dominated grid zones. Islands fall within water dominated UTM grid zones

(Fig. 11b), but it is almost possible to identify single buildings as well as open spaces in the urban layout. Besides, the probabilistic output seems to be highly related to the patterns of built-up areas suggesting that GHS-S2Net may be a proxy measure for building densities.

These examples provide experimental findings that support the GHS-S2Net model generalization capacity, which was already evidenced during the training phase (3.1.2). With a relatively small number of parameters $(1,447,042$ trainable parameters) and a very large number of samples $(511,502,073$ total number of built-up patches-See Supplementary material R1 for training samples per UTM zone), the model proved to be robust to noise or missing data with respect to the training sets, while effectively capturing the essential patterns and salient features, resulting in precise mapping of built-up areas.

\subsection{Validation of the model predictions and assessment of generalization performance}

Two approaches were implemented for the validation of the GHS-S2Net output that are based on comparison with independent cartographic data of building footprints, not employed for the training of the models:

- Continuous assessment: by testing the GHS-S2Net output as predictor of the built-up densities at the spatial resolution of $10 \mathrm{~m}$ through least-square linear regression; 
Fig. 9 Example predictions of GHS-S2Net in the form of probabilities of built-up areas. One example is given per each training set to demonstrate the benefit of the model output compared to the input best available training sets (a FB_HRS, b ESM_BU, c MS_BFP, d GHSL_BU)Google satellite imagery is used in the background
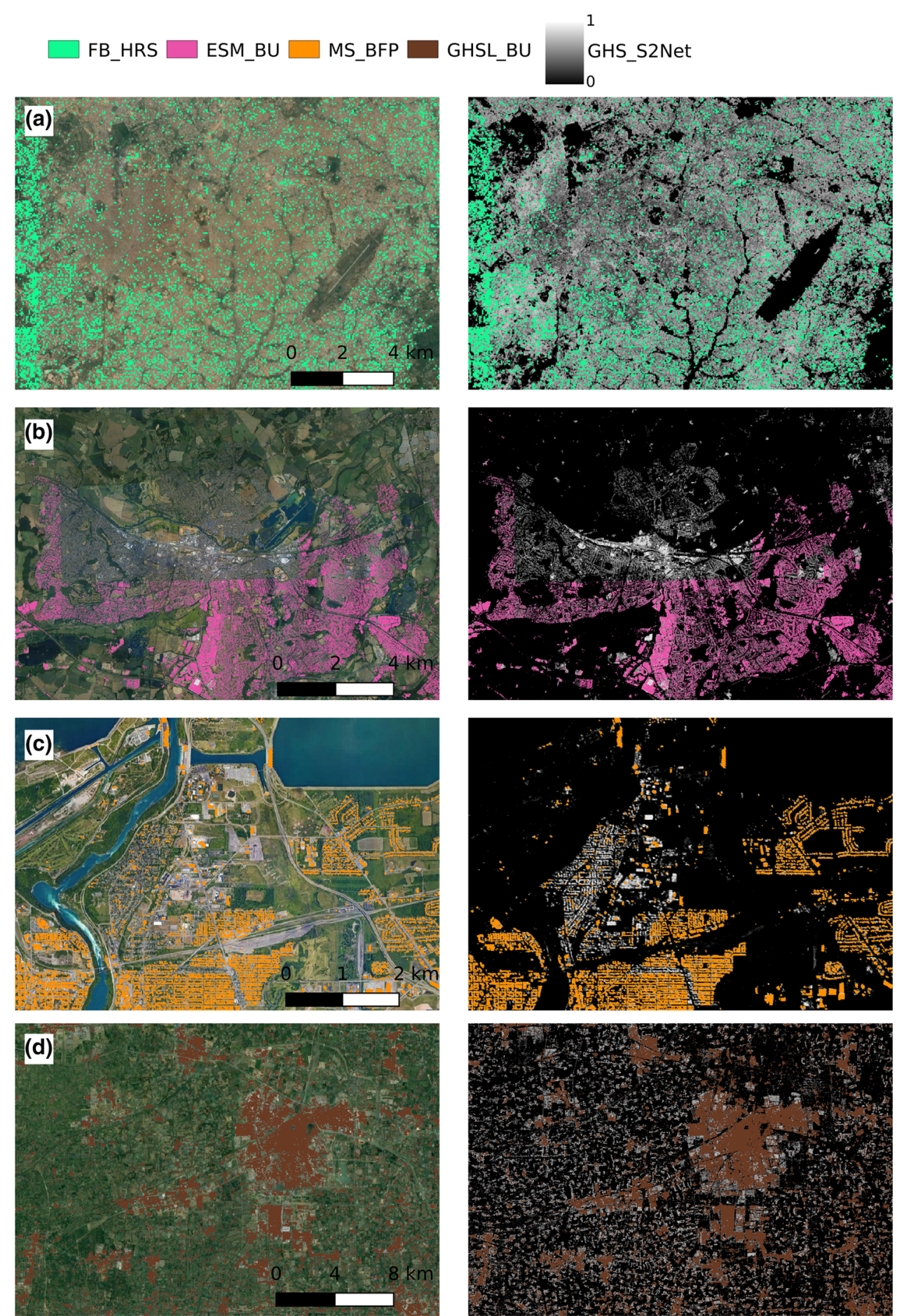

- Binary assessment: by evaluating the contingency table between the binarized outputs of GHS-S2net after the application of a probability cut-off value, and the binarized reference data used as a "ground-truth."

For the validation of pixel-wise predictions, a reference spatial database including single building delineation derived from digital cartography at a nominal scale of 1:10,000 was developed. The suitability of this database for the global scale validation of built-up products derived from remote sensing data has been previously evaluated in Corbane et al., 2019 [5]. The reference database consists of more than 40 million individual building polygons selected from 277 different areas of interest (AOI) around the globe. These are mostly local administrative units covering specific cities or full counties (for the United States of America) and spread across different continents. While not 

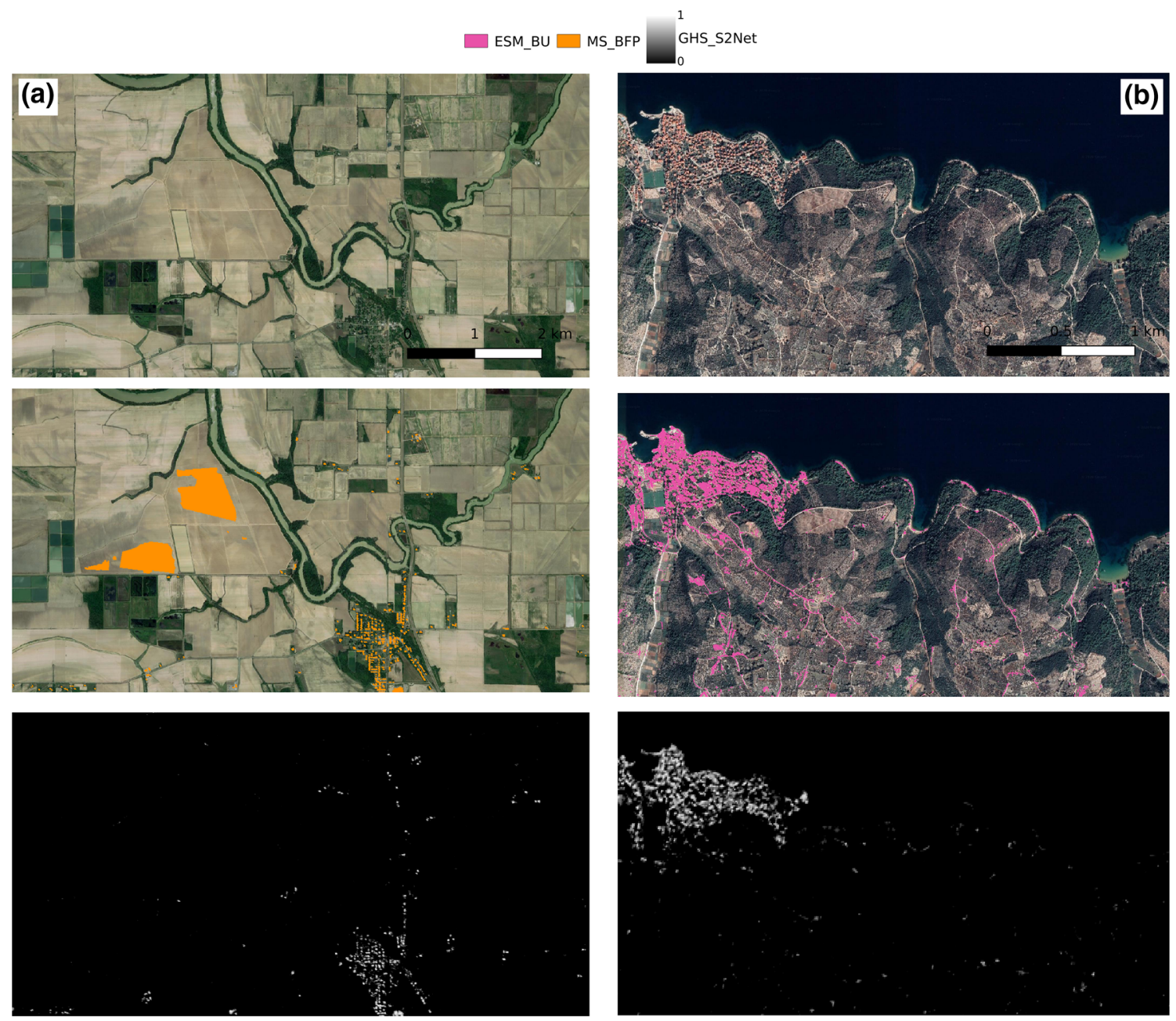

Fig. 10 Examples of reduction and mitigation of commission errors. The figures show a comparison between the probability of built-up areas obtained from the GHS-S2Net and the input training sets based

covering all the combinations of geographical, environmental, and cultural conditions that are determinant factors of the settlement patterns, the reference data spread across various landscapes. The reference years for the collected reference data range between 2012 and 2018 with the latter being the most frequent year of update. This makes the reference database suitable for the validation of the results derived from the Sentinel-2 pixel based image composite produced for the reference year 2018. The building footprints span over the whole spectrum of low-density and high-density human settlement patterns, representing typical rural, suburban and urban spatial patterns (see supplementary material R2 for more information on the spatial distribution and characteristics of the reference dataset). In order to support the accuracy assessment exercise, the reference data collected in vector format were converted into binary raster layers indicating the presence/absence of built-up areas. The rasterization of the vector cartographic on a MS_BFP in Northern America and b ESM_BU in EuropeGoogle satellite imagery is used in the background

data was performed at a spatial resolution of $10 \mathrm{~m}$ corresponding to the spatial resolution of the Sentinel-2 image composite and the outputs of the GHS-S2Net model.

\subsubsection{Continuous assessment: validation of the model output as predictor of built-up densities}

For analyzing the performance of the GHS-S2Net model as a predictor of the densities of built-up areas, we perform a regression analysis between the probability of built-up areas given by the model as response and the reference built-up surface densities as derived from the database of building footprints for the 277 different areas of interest. The knowledge of the systematic bias and gain parameters of the automatically classified built-up areas allows us to gain insights into the capacity of the GHS-S2Net model in capturing the patterns and densities of built-up areas and to identify a suitable threshold for the binarization of the 
Fig. 11 Example of refined built-up areas detection in the city of Sassari with different types and densities of buildings. a Extract from VHR Google imagery, b ESM_BU training set derived from Copernicus VHR_2015 and $\mathbf{c}$ output of GHS-S2Net representing probabilities in built-up areasGoogle satellite imagery is used in the background
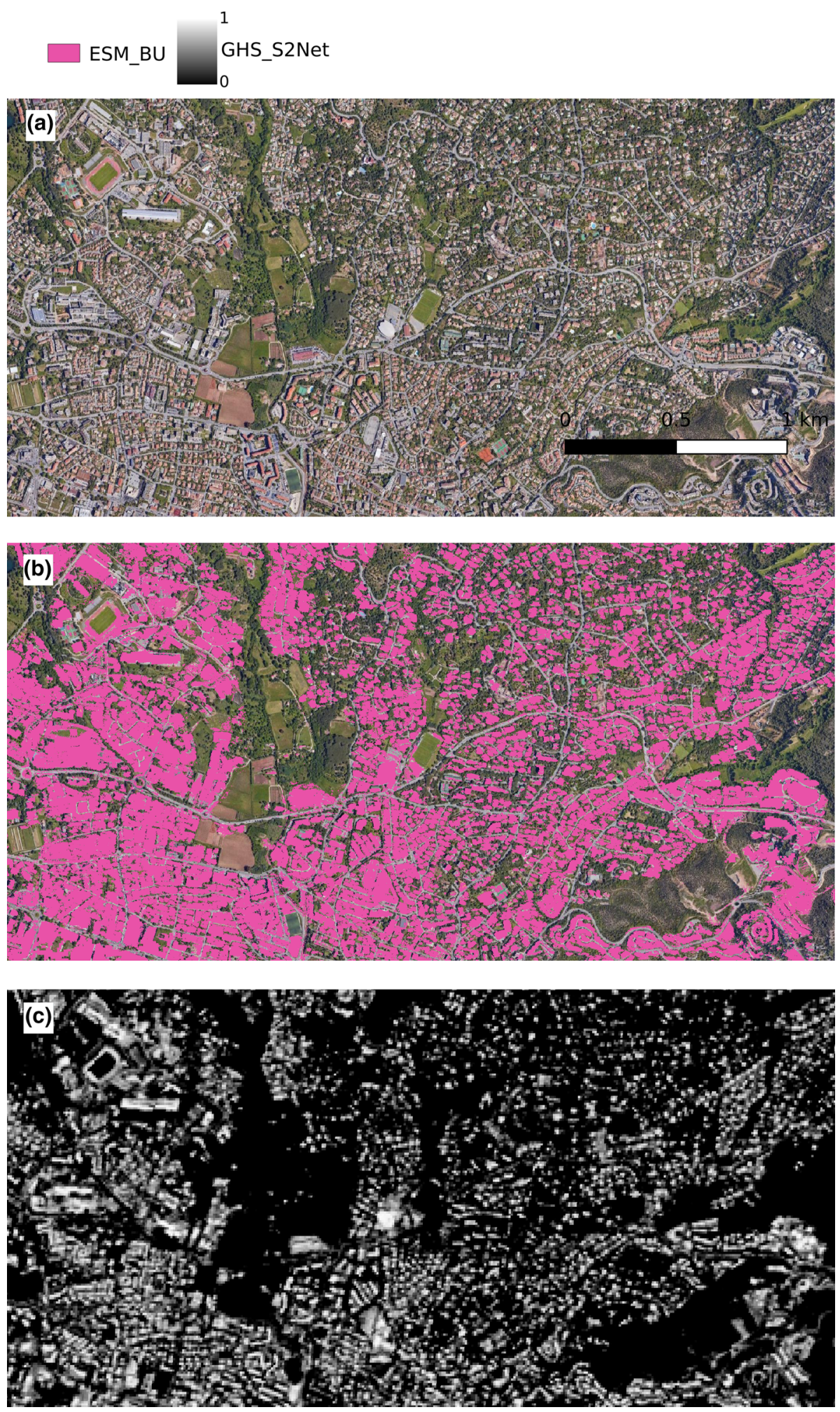

output probabilities for the subsequent accuracy assessment step.

The strength of the linear relation between the automatically generated built-up probabilities and the reference data is assessed through the Pearson correlation coefficient (r). The gain factor (slope) allows the user to model, retrofit and compare the results obtained from the GHS-S2Net model for the different AOIs. In addition, the slope of the regression is an indicator of the optimal threshold for 


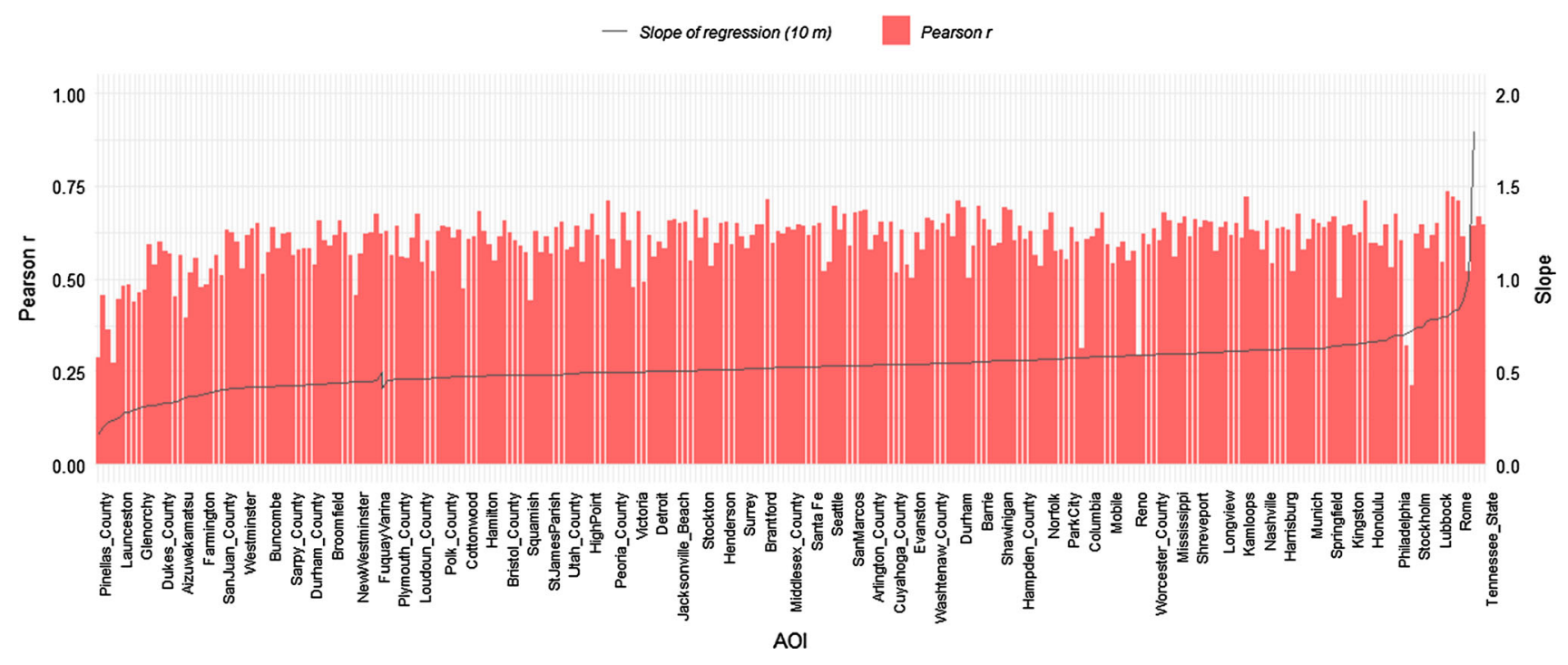

Fig. 12 Results of the regression analysis between the output probabilities of built-up areas and built-up densities at $10 \mathrm{~m}$ resolution. The results represented here by the correlation coefficient (r) and the slope of regression are shown for the 277 AOI

translating the built-up probabilities to binary values for the pixel-based accuracy assessment.

The results of the regression analysis at $10 \mathrm{~m}$ for all AOI sites showed an average correlation coefficient $\mathrm{r}$ of 0.67 and an average slope of 0.52 (Fig. 12).

The average correlation coefficient shows that the output probabilities from GHS-S2Net models are capable of capturing around $67 \%$ of the structural variability in built-up areas. The lowest correlation coefficients were observed for AOIs covering complete counties in the United States where there are a lot of building sizes below $100 \mathrm{~m}^{2}$ (which is the limit of the detectability of the Sentinel-2 sensor) and the built-up density is very low, less than $0.5 \%$. This is for instance the case of the Matanuska-Susitna Borough AOI which is a borough located in the state of Alaska, covering an area $9492.46 \mathrm{~km}^{2}$ with a built-up density of $0.1 \%$ and an average size of buildings of $140 \mathrm{~m}^{2}$ (Supplementary material R2). The output probabilities of the GHS-S2Net models seem to better capture building densities in urban areas and high density AOIs where the correlation coefficients were greater than 0.6. This is the case for example of the AOI covering San Francisco city with an area of 194 $\mathrm{km}^{2}$ and a building density of $26.4 \%$.

It is also worth noting that the gain factor (slope) translating the built-up probabilities as derived from Sentinel-2 data to built-up surface densities as derived from the reference cartographic data is almost constant. The slope has an average of 0.2 in low density AOIs, in particular those covering full counties in the United States (e.g., San Juan County). In high-density AOIs covering cities, the slope (bias) is higher (e.g., city of Rome where the slope is close to 0.8 ) with an average around 0.54 .
According to these findings, it is not straightforward to define one general-purpose threshold to binarize the output of the GHS-S2Net models into two classes "built-up" and "non-built-up." A threshold of 0.2 would then be good compromise targeting large areas including scattered settlement patterns, in particular rural areas, while a more conservative threshold of 0.5 would be more suitable for areas largely dominated by high-density built-up areas (i.e. city centers). Following this finding, both thresholds were applied to the outputs of the GHS-S2Net models for assessing the quality of the classifications following a pixel-wise accuracy method.

\subsubsection{Binary accuracy assessment}

The thresholds 0.2 and 0.5 identified in the previous regression analysis were used to binarize the probabilistic output as required by the pixel-wise binary accuracy assessment at the spatial resolution of the sensor. Standard accuracy and error metrics derived from the confusion matrix were calculated for the binary results obtained with the two thresholds. Given the lack of a single universally accepted measure of agreement, we use a combination of two main performance metrics to give a complete picture of the performance of the GHS-S2Net models: the balanced accuracy and the Kappa coefficient that were introduced to the remote sensing community and recommended by Congalton, 2011 [87]. The Balanced Accuracy and Kappa are measures of classification accuracy, the former providing information about the rate of correctly classified pixels in an unbalanced setting where non-built-up pixels are predominant compared to built-up pixels. The latter compensates for random chance in the pixels assignment. 
Probability_thresh_0.2 审 Probability_thresh_0.5

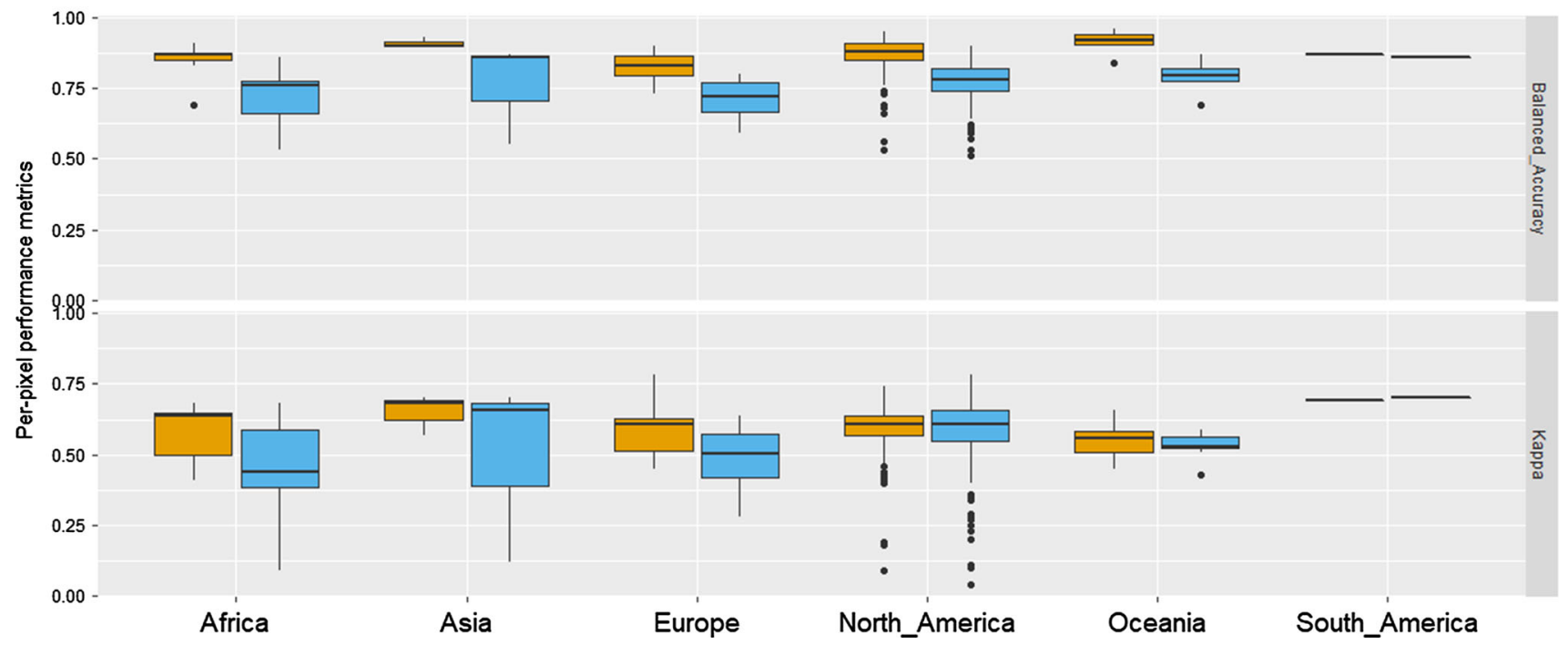

Fig. 13 Per-continent, box plots of the performance metrics (Balanced Accuracy and Kappa) of the two binary classification outputs obtained by applying 0.2 and 0.5 thresholds to the probability outputs

The results of the per-pixel accuracy assessment with the two binary outputs are summarized in Fig. 13 and disaggregated per continent. The figure shows the average and standard deviations of the Balanced Accuracy and Kappa coefficients per binary output and per continent. The 277 AOI were grouped by continent to evidence major improvements, especially in areas where previous global products failed to produce satisfactory results. Overall, both binary classifications produce good results with an average Balanced Accuracy greater than 0.7 and an average Kappa greater than 0.5. However, when compared to the binary outputs derived with the 0.5 probability threshold, the classification with a less conservative threshold of 0.2 produces better agreement with the reference data, consistently for all continents. The best results in the least conservative classification outputs (threshold of 0.2) were obtained in Oceania an Asia with an average Balanced Accuracy of 0.91, followed by North America and Africa where the mean Balanced Accuracies were equal to 0.86 and 0.85 , respectively.

The results of the per-pixel accuracy assessment, in particular those obtained by applying a low threshold to the probability outputs, constitute a strong evidence of the modeling power of the GHS-S2Net and the reliability of the outputs. They are also a confirmation of the merit of the new classification framework for identifying settlements in challenging landscapes such as in Africa and Asia. They also suggest that for the generation of a global binary classification from the probabilistic output of the models, a low probability threshold is recommended, in particular if the purpose is to capture all the scattered settlements in rural landscapes such as in Africa. In this particular context, the binary outputs obtained with a threshold of 0.2 outperform significantly those derived from the conservative threshold.

\subsection{Comparison between the results of close range and far range transfer learning}

When computing the GHS-S2Net predictions at the global scale, the majority of the UTM grid zones and in particular the $100 \times 100 \mathrm{~km}^{2}$ tiles were processed with the close range transfer learning. However, to allay the scarcity and quality issues in the training dataset, 28 UTM grid zones were classified according to the far range transfer learning and the outputs were compared to those obtained by the direct close range transfer learning. Figure 14a illustrates the differences between close range (middle figure) and far range transfer learning (bottom figure) in areas suffering from the lack of training samples (e.g., in Ethiopia). It shows the capacity of the far range transfer learning in discovering undetected built-up features in UTM grid zone 37P, on the basis of the parameters of the model trained in the neighboring UTM grid zone $37 \mathrm{M}$. In such a situation, the close range transfer learning was less effective in identifying those scattered settlements due to insufficient training samples in the UTM grid zone 37P.

Figure 14b shows another example with respect to the city of Moscow, showing the added-value of the far range transfer learning in areas where only the GHS_BU lowresolution training data were available (UTM grid zone 37U). The example highlights the generalization capacity of the GHS-S2Net trained on a UTM grid zone where detailed training samples are available (e.g., in UTM grid 

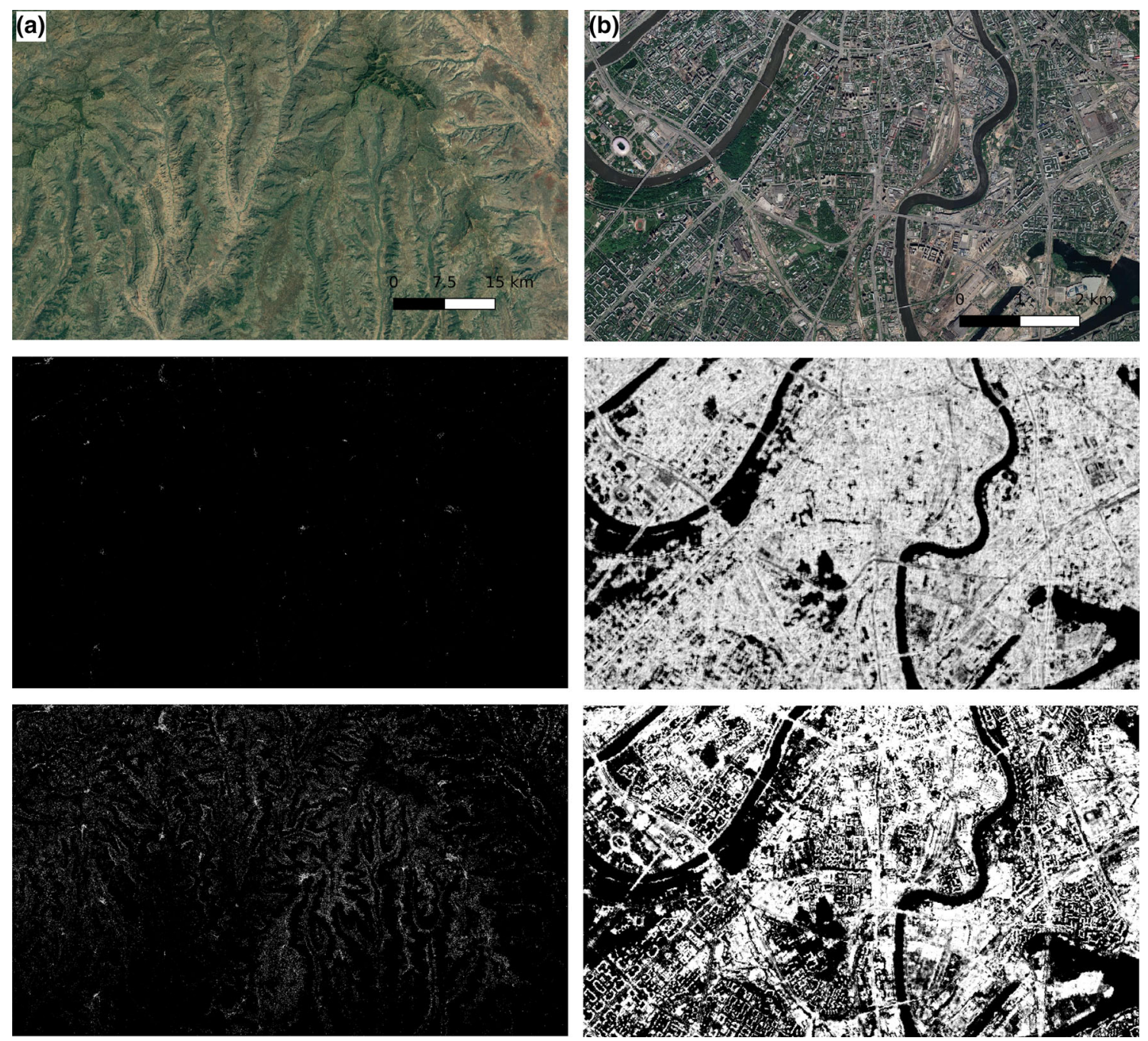

Fig. 14 Comparative results of the close range (middle figures) and far range transfer learning (bottom figures) in a Ethiopia, b Moscow (Russia)-Google satellite imagery is used in the background

zone $34 \mathrm{U}$ ) and then applied to the nearby zone. The generalization capacity of the model here is reflected in: (i) reproducing fine-scale settlement structures in dense built-up areas, (ii) reducing overdetections of roads and other impervious features and (iii) enhancing the sharp delineation of buildings and open spaces in the built-up areas.

Moscow is one of the cities where detailed building footprints were available in the reference database used in the validation exercise. The availability of "ground-truth" data enabled to conduct a quantitative binary accuracy assessment of the results of far range transfer learning in comparison with those obtained with the close range transfer learning. The results are illustrated in Table 2 for the binary outputs with cut-off values of 0.2 and 0.5 . They show higher overall and balanced accuracy values resulting from the application of far range transfer leaning. These results are an additional evidence of the enhanced mapping capabilities of a well-designed far range transfer learning approach deployed in this work.

The encouraging results were determinant for expanding the application of far range transfer learning which was
Table 2 Summary Results of binary accuracy assessment of the close range and far transfer learning in the city of Moscow based on detailed building footprints

\begin{tabular}{|c|c|c|c|c|}
\hline & \multicolumn{2}{|c|}{ Overall accuracy } & \multicolumn{2}{|c|}{ Balanced accuracy } \\
\hline & 0.2 cut-off & 0.5 cut-off & 0.2 cut-off & 0.5 cut-off \\
\hline Close range transfer learning & 0.61 & 0.67 & 0.75 & 0.76 \\
\hline Far range transfer learning & 0.77 & 0.83 & 0.81 & 0.78 \\
\hline
\end{tabular}


finally implemented on a total of 28 UTM grid zones. The selection of source and target UTM grid zones was mainly driven by spatial adjacency or similarities in the landscape and in the type of built-up areas.

\section{Discussion and future work}

In this paper, we presented a novel end-to-end framework for large-scale pixel-wise classification of built-up areas from high-resolution satellite imagery. The developed multi-model approach designated by GHS-S2Net builds on a relatively simple $\mathrm{CNN}$ architecture. The implementation of the models on a global cloud-free Sentinel-2 image composite provides the most detailed and complete map reporting about built-up areas in the form of probability outputs (i.e., probability of a pixel to belong to the class 'built-up'). The results confirm the high generalization capacity of the model and its ability to not only detect new built-up areas in difficult landscapes (i.e., in Africa and Asia) without site-specific training sets, but also its potential to mitigate commission errors in the best available training sets reporting about built-up areas across the globe.

The implementation of the developed framework for large classification of human settlements was achieved thanks to three main building blocks:

- The multi-neuro modeling methodology, which follows the UTM grid zones schema and the systematic sampling within each UTM grid zone. This approach of training multiple lightweight models at global scale allows decomposing the optimization phase into smaller tasks, which are then solved in parallel. The adopted sampling approach meets the three following criteria: class balance, diversity, and representativeness. It shows to be suitable for an optimal learning of the models at a global scale without compromising performance;

- The transfer learning includes both the close range and the far range transfer learning. Both approaches benefit from parameter-based transfer methods where the optimal parameters found in the source domain classifier are used for the target domain. The novelty of the approach implemented in the paper was the use of the close range transfer learning within the same UTM grid zone in a way to alleviate the computational burden and avoid overfitting issues. The far range transfer learning leverages the optimal parameters found when training the models with detailed and high-quality training sets in a given UTM grid zone and then applying them to neighboring zones subject to training data scarcity. The far range transfer learning allowed allaying the scarcity and quality issues in the training sets while achieving outstanding performance in the reduction of commission and omission errors found in the best available data and in the refinement of built-up areas detection;

- The deployment of the high-throughput processing, including data preparation, learning and inference on the multi-petabyte scale JEODPP platform. The big data multi GPU platform enables: (i) the efficient storage of the large volume of input satellite data (15 TB) and the output (1.5 TB) maps encoded in 16 bits, (ii) the parallel training of the models on an heterogeneous cluster of GPUs, and the (iii) optimal load balance in terms of data retrieval and processing from and to the distributed system due to the efficient co-location of the data with the processing units.

The validation of the results with an independent reference dataset of building footprints covering 277 sites across the world establishes the reliability of the built-up layer produced by the GHS-S2Net approach and the model robustness against both the variable conditions in the satellite imagery and the heterogeneity in the landscapes and built-up characteristics. The most noticeable achievement is the capacity of the model to classify built-up areas in remote areas (e.g., in Africa and in Asia), reported in none of the global products (i.e., GUF, WSF, FROM GLC10). Further research is currently being deployed to quantitatively assess the improvements gained with the GHS-S2Net compared to other global products reporting on built-up areas.

Another significant result is the strong relationship between the output probabilities and the building densities suggesting that the model outputs can be used as proxy measures for building densities without additional calibration or modeling.

Despite the unprecedented results obtained by the proposed approach on an extremely challenging dataset in terms of spatial coverage, resolution and spectral variability, some challenges need to be considered, especially if the aim is to regularly update the built-up layer for continuous monitoring of human settlements with Copernicus Sentinel-2 data. The challenges pertain to methodological choices when designing the model and during its scaling to the classification of the global composite:

- The choice of patch size: in general, assessment of CNN accuracy indicates that using larger patch sizes yields higher accuracies because the network is able to learn more contextual features. In the case of the Sentinel-2 pixel-based classification, the experiments performed by [71] on Sentinel-2 data showed that larger patch sizes (e.g., $15 \times 15)$ did not yet yield significant improvement in the model accuracy. In this work, we tested a $10 \times 10$ patch size resulting in a deeper network topology, yet the loss function did not improve 
during the training phase whereas the prediction accuracy worsened.

- The far range transfer learning: the strategy for implementing the far range transfer learning was based on criteria related to spatial adjacency of UTM grid zones or similarities in the landscape and in the type of built-up areas. The potential of this approach for mitigating problems in the training data and for deriving fine-grained classification outputs was clearly demonstrated in the classification results. Nevertheless, the added-value of this approach was not fully exploited in the context of this work. Additional work should focus on the analysis of spatial patterns of landscape features and typologies of built-up areas and their influence on the outputs of the classification with $G H S$ S2Net. The ultimate goal is to unveil the underlying rules and associations for designing a more systematic approach to identify the source and the target UTM grid zones candidate for the far range transfer learning.

- The variable quality of the training data: despite their outstanding learning capability, the lack of accurate training data might limit the applicability of CNN models in realistic remote-sensing contexts [88]. For our global scale application, the strategy was to collect the best publicly available training data and reporting about built-up areas. The higher the spatial resolution of the training data, the more detailed is the output of the classification. Ideally, the spatial resolution of the input training data should be equal or better to that of the input Sentinel-2 imagery. As described in Sect. 2.2, the reference data sources have variable spatial resolutions. In addition, the trustworthiness of samples is highly variable across the different sources but also within the same reference data source. The lack of consistency in the training data produces outputs with variable qualities depending on the input data used for training the models. This was reflected by the results of the validation when disaggregated per continent. One approach to deal with imperfect training data was to use the far range transfer learning. However, this approach has a limited applicability at global scale since it supposes that the target UTM grid zones have similar characteristics (in terms of landscape and types of built-up areas) with the source zones. Another approach is to use a two-step training approach in which the models are first initialized by using a large amount of possibly inaccurate reference data, and then refined on a small amount of accurately labeled data, similarly to the method developed in Maggiori et al. [88]. In the context of our large-scale classification, it is perfectly reasonable to use the output produced by the GHS-S2Net to train a new model. The use of high quality and consistent outputs produced for the reference year 2018 by the application of the $G H S$ S2Net model at global scale is a key for frequent updates of built-up layers from Sentinel-2 Copernicus data and for continuous monitoring of built-up areas.

The outputs of this work are an additional demonstration of the far-reaching opportunities of using DL in remote sensing applications. They set the path to a new wave of promising research to tackle unprecedented large-scale challenges in urban related applications building on remote sensing data. Further research is currently being carried out to explore the potentials of using DL and EO to assess changes in built-up areas and to generate consistent time series reporting about the dynamics of human settlements at the European and global scales. Characterization of the urban environment is another research field in which DL and EO are bringing new advances and a concrete opportunity to develop models for describing the buildings' typology and the morphology of the cities, hence addressing global interrelated challenges of urbanization and climate change.

Availability of data and material The final map of built-up areas has been uploaded in Google Earth Engine for visualization. The values for the probabilities have been rescaled to 8 bits in the range $0-100$ with no data values set to 255 . https://code.earthengine.google.com/ 6a1457205bd295a44902a6c2eb266204?hideCode=true.

\section{Compliance with ethical standards}

Conflict of interest The authors have no competing interests.

Open Access This article is licensed under a Creative Commons Attribution 4.0 International License, which permits use, sharing, adaptation, distribution and reproduction in any medium or format, as long as you give appropriate credit to the original author(s) and the source, provide a link to the Creative Commons licence, and indicate if changes were made. The images or other third party material in this article are included in the article's Creative Commons licence, unless indicated otherwise in a credit line to the material. If material is not included in the article's Creative Commons licence and your intended use is not permitted by statutory regulation or exceeds the permitted use, you will need to obtain permission directly from the copyright holder. To view a copy of this licence, visit http://creativecommons. org/licenses/by/4.0/.

\section{References}

1. Melchiorri M, Florczyk A, Freire S, Schiavina M, Pesaresi M, Kemper T (2018) Unveiling 25 years of planetary urbanization with remote sensing: perspectives from the global human settlement layer. Remote Sens 10(5):768. https://doi.org/10.3390/ rs 10050768

2. Zhu Z et al (2019) Understanding an urbanizing planet: strategic directions for remote sensing. Remote Sens Environ 228:164-182. https://doi.org/10.1016/j.rse.2019.04.020 
3. Esch $T$ et al (2017) Breaking new ground in mapping human settlements from space-the global urban footprint. ISPRS J Photogramm Remote Sens 134:30-42. https://doi.org/10.1016/j. isprsjprs.2017.10.012

4. Corbane $\mathrm{C}$ et al (2017) Big earth data analytics on Sentinel-1 and Landsat imagery in support to global human settlements mapping. Big Earth Data 1(1-2):118-144. https://doi.org/10.1080/ 20964471.2017.1397899

5. Corbane C et al (2019) Automated global delineation of human settlements from 40 years of Landsat satellite data archives. Big Earth Data 3(2):140-169. https://doi.org/10.1080/20964471. 2019.1625528

6. Marconcini M, Metz-Marconcini A, Üreyen S et al (2020) Outlining where humans live, the World settlement footprint 2015. Sci Data 7:242. https://doi.org/10.1038/s41597-020-00580-5

7. Gong $P$ et al (2019) Stable classification with limited sample: transferring a $30-\mathrm{m}$ resolution sample set collected in 2015 to mapping 10-m resolution global land cover in 2017. Sci Bull 64(6):370-373

8. Deng C, Wu C (2012) BCI: A biophysical composition index for remote sensing of urban environments. Remote Sens Environ 127:247-259. https://doi.org/10.1016/j.rse.2012.09.009

9. Sun Z, Wang C, Guo H, Shang R (2017) A modified normalized difference impervious surface index (MNDISI) for automatic urban mapping from landsat imagery. Remote Sens 9(9):942. https://doi.org/10.3390/rs9090942

10. Gong $P$ et al (2020) Annual maps of global artificial impervious area (GAIA) between 1985 and 2018. Remote Sens Environ 236:111510. https://doi.org/10.1016/j.rse.2019.111510

11. Wu C, Murray AT (2003) Estimating impervious surface distribution by spectral mixture analysis. Remote Sens Environ 84(4):493-505. https://doi.org/10.1016/S0034-4257(02)00136-0

12. Li G, Lu D, Moran E, Hetrick S (2013) Mapping impervious surface area in the Brazilian Amazon using Landsat Imagery. GIScience Remote Sens 50(2):172-183. https://doi.org/10.1080/ 15481603.2013.780452

13. Pesaresi M, Benediktsson JA (2001) A new approach for the morphological segmentation of high-resolution satellite imagery. Geosci Remote Sens IEEE Trans On 39(2):309-320. https://doi. org/10.1109/36.905239

14. Esch T, Thiel M, Schenk A, Roth A, Muller A, Dech S (2010) Delineation of urban footprints From TerraSAR-X data by analyzing speckle characteristics and intensity information. IEEE Trans Geosci Remote Sens 48(2):905-916. https://doi.org/10. 1109/TGRS.2009.2037144

15. Goldewijk KK (2001) Estimating global land use change over the past 300 years: the HYDE database. Global Biogeochem Cycles 15(2):417-433

16. Elvidge CD et al (2007) Global distribution and density of constructed impervious surfaces. Sensors 7:1962-1979

17. Yang L, Huang C, Homer CG, Wylie BK, Coan MJ (2003) An approach for mapping large-area impervious surfaces: synergistic use of Landsat-7 ETM+ and high spatial resolution imagery. Can J Remote Sens 29(2):230-240. https://doi.org/10.5589/m02-098

18. Schneider A, Friedl MA, Potere D (2010) Mapping global urban areas using MODIS 500-m data: New methods and datasets based on 'urban ecoregions.' Remote Sens Environ 114:1733-1746. https://doi.org/10.1016/j.rse.2010.03.003

19. Goldblatt R et al (2018) Using Landsat and nighttime lights for supervised pixel-based image classification of urban land cover. Remote Sens Environ 205:253-275

20. X. Zhang et al. (2020) Development of a global 30-m impervious surface map using multi-source and multi-temporal remote sensing datasets with the Google Earth Engine platform. Antroposhere-Land Cover and Land Use, preprint, Jan. 2020. https:// doi.org/10.5194/essd-2019-200.
21. Ban Y, Gong P, Giri C (2015) Global land cover mapping using earth observation satellite data: recent progresses and challenges. ISPRS J Photogramm Remote Sens 103:1-6. https://doi.org/10. 1016/j.isprsjprs.2015.01.001

22. DeFries R, Hansen M, Townshend J (1995) Global discrimination of land cover types from metrics derived from AVHRR pathfinder data. Remote Sens Environ 54(3):209-222. https://doi.org/10. 1016/0034-4257(95)00142-5

23. Potere D, Schneider A, Angel S, Civco DL (2009) Mapping urban areas on a global scale: which of the eight maps now available is more accurate? Int J Remote Sens 30:6531-6558

24. Florczyk AJ et al (2019) The generalised settlement area: mapping the earth surface in the vicinity of built-up areas. Int J Digit Earth. https://doi.org/10.1080/17538947.2018.1550121

25. Schneider A, Woodcock CE (2008) Compact, dispersed, fragmented, extensive? a comparison of urban growth in twenty-five global cities using remotely sensed data, pattern metrics and census information. Urban Stud 45(3):659-692. https://doi.org/ 10.1177/0042098007087340

26. Sharma RC, Tateishi R, Hara K, Gharechelou S, Iizuka K (2016) Global mapping of urban built-up areas of year 2014 by combining MODIS multispectral data with VIIRS nighttime light data. Int J Digit Earth 9(10):1004-1020. https://doi.org/10.1080/ 17538947.2016.1168879

27. Leyk S, Uhl JH, Balk D, Jones B (2018) Assessing the accuracy of multi-temporal built-up land layers across rural-urban trajectories in the United States. Remote Sens Environ 204:898-917. https://doi.org/10.1016/j.rse.2017.08.035

28. Defries R, Townshend J (1999) Global land cover characterization from satellite data: from research to operational implementation? GCTE/LUCC Research Review. Global Ecol Biogeogr 8(5):367-379

29. Yang H, Li S, Chen J, Zhang X, Xu S (2017) The standardization and harmonization of land cover classification systems towards harmonized datasets: a review. ISPRS Int J Geo-Inf 6(5):154. https://doi.org/10.3390/ijgi6050154

30. Corbane C et al. (2019) Automatic Image Data Analytics from a Global Sentinel-2 Composite for the Study of Human Settlements. presented at the Big Data from Space (BiDS'19), https:// doi.org/10.2760/848593.

31. Pesaresi M, Corbane C, Julea A, Florczyk A, Syrris V, Soille P (2016) Assessment of the added-value of sentinel-2 for detecting built-up areas. Remote Sens 8(4):299. https://doi.org/10.3390/ rs8040299

32. Ma L, Liu Y, Zhang X, Ye Y, Yin G, Johnson BA (2019) Deep learning in remote sensing applications: A meta-analysis and review. ISPRS J Photogramm Remote Sens 152:166-177. https:// doi.org/10.1016/j.isprsjprs.2019.04.015

33. Wang S, Quan D, Liang X, Ning M, Guo Y, Jiao L (2018) A deep learning framework for remote sensing image registration. ISPRS J Photogramm Remote Sens 145:148-164. https://doi.org/10. 1016/j.isprsjprs.2017.12.012

34. Tan W, Xiang P, Zhang J, Zhou H, Qin H (2020) Remote sensing image fusion via boundary measured dual-channel PCNN in multi-scale morphological gradient domain. IEEE Access 8:42540-42549. https://doi.org/10.1109/ACCESS.2020.2977299

35. Huang W, Xiao L, Wei Z, Liu H, Tang S (2015) A new pansharpening method with deep neural networks. IEEE Geosci Remote Sens Lett 12(5):1037-1041. https://doi.org/10.1109/ LGRS.2014.2376034

36. Cai Y et al (2018) A high-performance and in-season classification system of field-level crop types using time-series Landsat data and a machine learning approach. Remote Sens Environ 210:35-47. https://doi.org/10.1016/j.rse.2018.02.045

37. Cheng G, Zhou P, Han J (2016) Learning rotation-invariant convolutional neural networks for object detection in VHR 
optical remote sensing images. IEEE Trans Geosci Remote Sens 54(12):7405-7415. https://doi.org/10.1109/TGRS.2016.2601622

38. Ball JE, Anderson DT, Chan CS (2017) Comprehensive survey of deep learning in remote sensing: theories, tools, and challenges for the community. J Appl Remote Sens 11(04):1. https://doi.org/ 10.1117/1.JRS.11.042609

39. Zhang L, Xia G-S, Wu T, Lin L, Tai XC (2016) Deep learning for remote sensing image understanding. J Sens 2016:1-2. https:// doi.org/10.1155/2016/7954154

40. Kussul N, Lavreniuk M, Skakun S, Shelestov A (2017) Deep learning classification of land cover and crop types using remote sensing data. IEEE Geosci Remote Sens Lett 14(5):778-782. https://doi.org/10.1109/LGRS.2017.2681128

41. Chen Y, Jiang H, Li C, Jia X, Ghamisi P (2016) Deep feature extraction and classification of hyperspectral images based on convolutional neural networks. IEEE Trans Geosci Remote Sens 54(10):6232-6251. https://doi.org/10.1109/TGRS.2016.2584107

42. Ishii $T$, Nakamura $R$, Nakada $H$, Mochizuki $Y$, Ishikawa $H$ (2015) Surface object recognition with CNN and SVM in Landsat 8 images. In: 2015 14th IAPR International Conference on Machine Vision Applications (MVA), Tokyo, Japan, May 2015, pp. 341-344, https://doi.org/10.1109/MVA.2015.7153200.

43. Chen C, Ma Y, Ren G (2019) A convolutional neural network with fletcher-reeves algorithm for hyperspectral image classification. Remote Sens 11(11):1325. https://doi.org/10.3390/ rs11111325

44. Geng J, Wang H, Fan J, Ma X (2017) Deep supervised and contractive neural network for SAR image classification. IEEE Trans Geosci Remote Sens 55(4):2442-2459. https://doi.org/10. 1109/TGRS.2016.2645226

45. Fang B, Pan L, Kou R (2019) Dual learning-based siamese framework for change detection using Bi-temporal VHR optical remote sensing images. Remote Sens 11(11):1292. https://doi. org/10.3390/rs11111292

46. Hutchison D et al (2010) Learning to detect roads in high-resolution aerial images. In: Daniilidis K, Maragos P, Paragios N (eds) Computer Vision-ECCV 2010, vol 6316. Springer, Berlin Heidelberg, pp 210-223

47. Yang J, Ji L, Geng X, Yang X, Zhao Y (2019) Building detection in high spatial resolution remote sensing imagery with the U-Rotation Detection Network. Int J Remote Sens 40(15):6036-6058. https://doi.org/10.1080/01431161.2019. 1587200

48. Huang B, Zhao B, Song Y (2018) Urban land-use mapping using a deep convolutional neural network with high spatial resolution multispectral remote sensing imagery. Remote Sens Environ 214:73-86. https://doi.org/10.1016/j.rse.2018.04.050

49. Romero A, Gatta C, Camps-Valls G (2016) Unsupervised deep feature extraction for remote sensing image classification. IEEE Trans Geosci Remote Sens 54(3):1349-1362. https://doi.org/10. 1109/TGRS.2015.2478379

50. Yang W, Yin X, Xia G-S (2015) Learning high-level features for satellite image classification with limited labeled samples. IEEE Trans Geosci Remote Sens 53(8):4472-4482. https://doi.org/10. 1109/TGRS.2015.2400449

51. Farabet C, Couprie C, Najman L, LeCun Y (2013) Learning hierarchical features for scene labeling. IEEE Trans Pattern Anal Mach Intell 35(8):1915-1929. https://doi.org/10.1109/TPAMI. 2012.231

52. Casasent DP, Neiberg LM (1995) Classifier and shift-invariant automatic target recognition neural networks. Neural Netw 8(7-8):1117-1129

53. Li W, Liu H, Wang Y, Li Z, Jia Y, Gui G (2019) deep learningbased classification methods for remote sensing images in urban built-up areas. IEEE Access 7:36274-36284. https://doi.org/10. 1109/ACCESS.2019.2903127
54. Tian T, Li C, Xu J, Ma J (2018) Urban area detection in very high resolution remote sensing images using deep convolutional neural networks. Sensors 18(3):904. https://doi.org/10.3390/s18030904

55. Stiller D, Stark T, Wurm M, Dech S, Taubenbock H (2019) Large-scale building extraction in very high-resolution aerial imagery using Mask R-CNN. In: 2019 Joint Urban Remote Sensing Event (JURSE), Vannes, France, May 2019, pp. 1-4, https://doi.org/10.1109/JURSE.2019.8808977.

56. Bramhe VS, Ghosh SK, Garg PK (2018) Extraction Of built-up areas using convolutional neural networks and transfer learning from sentinel-2 satellite images. ISPRS Int Arch Photogramm Remote Sens Spat Inf Sci XLII-3:79-85. https://doi.org/10.5194/ isprs-archives-XLII-3-79-2018

57. Qiu C, Schmitt M, Geiß C, Chen T-HK, Zhu XX (2020) A framework for large-scale mapping of human settlement extent from Sentinel-2 images via fully convolutional neural networks. ISPRS J Photogramm Remote Sens 163:152-170. https://doi.org/ 10.1016/j.isprsjprs.2020.01.028

58. Soille $\mathrm{P}$ et al (2017) A versatile data-intensive computing platform for information retrieval from big geospatial data. Future Gener Comput Syst. https://doi.org/10.1016/j.future.2017.11.007

59. White JC et al (2014) Pixel-based image compositing for largearea dense time series applications and science. Can J Remote Sens 40(3):192-212. https://doi.org/10.1080/07038992.2014. 945827

60. Griffiths P, van der Linden S, Kuemmerle T, Hostert P (2013) A Pixel-based landsat compositing algorithm for large area land cover mapping. IEEE J Sel Top Appl Earth Obs Remote Sens. 6(5):2088-2101. https://doi.org/10.1109/JSTARS.2012.2228167

61. Gorelick N, Hancher M, Dixon M, Ilyushchenko S, Thau D, Moore R (2017) Google earth engine: planetary-scale geospatial analysis for everyone. Remote Sens Environ. https://doi.org/10. 1016/j.rse.2017.06.031

62. Corbane C, Politis P, Kempeneers P, Simonetti D, Soille P, Burger A, Pesaresi M, Sabo F, Syrris V, Kemper T (2020) A global cloud free pixel-based image composite from Sentinel-2 data. Data Brief 31:105737. https://doi.org/10.1016/j.dib.2020. 105737

63. Corbane C, Politis P (2020) GHS-composite-S2 R2020A - Sentinel-2 global pixel based image composite from L1C data for the period 2017-2018. European Commission, Joint Research Centre (JRC), Apr. 15, 2020, https://doi.org/10.2905/0BD1DFAB-E3114046-8911-C54A8750DF79.

64. Pesaresi M, Syrris V, Julea A (2016) A new method for earth observation data analytics based on symbolic machine learning. Remote Sens 8(5):399. https://doi.org/10.3390/rs8050399

65. Corban C, Sabo F, Syrris V, Kemper T, Politis P, Pesaresi M, Soille P, Osé K (2020) Application of the symbolic machine learning to copernicus VHR imagery: the European settlement map. IEEE Geosci Remote Sens Lett 17(7):1153-1157

66. "Facebook High Resolution Settlement," 2019. https://ai.face book.com/blog/mapping-the-world-to-help-aid-workers-withweakly-semi-supervised-learning/ (accessed Feb. 13, 2020).

67. "Facebook's Data for Good program," Facebook's Data for Good program, 2020. https://data.humdata.org/organization/face book (accessed Feb. 13, 2020).

68. Tiecke TG et al (2017) Mapping the world population one building at a time. ArXiv Prepr. ArXiv171205839

69. "Microsoft Building Footprints," Microsoft Releases 125 million Building Footprints in the US as Open Data, 2018. https://blogs. bing.com/maps/2018-06/microsoft-releases-125-million-build ing-footprints-in-the-us-as-open-data.

70. Pesaresi M et al (2013) A global human settlement layer from optical HR/VHR RS data: concept and first results. IEEE J Sel Top Appl Earth Obs Remote Sens 6(5):2102-2131. https://doi. org/10.1109/JSTARS.2013.2271445 
71. Syrris V, Hasenohr P, Delipetrev B, Kotsev A, Kempeneers P, Soille P (2019) Evaluation of the potential of convolutional neural networks and random forests for multi-class segmentation of sentinel-2 imagery. Remote Sens 11:907. https://doi.org/10. 3390/rs11080907

72. Simonyan K, Zisserman A (2014) Very Deep Convolutional Networks for Large-Scale Image Recognition. CoRR, vol. abs/ 1409.1556, 2014, [Online]. Available: https://arxiv.org/abs/1409. 1556.

73. Szegedy C et al (2014) Going Deeper with Convolutions. CoRR, vol. abs/1409.4842, [Online]. Available: https://arxiv.org/abs/ 1409.4842.

74. Hochreiter S, Bengio Y, Frasconi P, Schmidhuber J (2001) Gradient flow in recurrent nets: the difficulty of learning longterm dependencies. In: Kremer SC, Kolen JF (eds) A field guide to dynamical recurrent neural networks. IEEE Press, New York

75. Nwankpa C, Ijomah W, Gachagan A, Marshall S (2018) Activation Functions: Comparison of trends in Practice and Research for Deep Learning. ArXiv, vol. abs/1811.03378, 2018.

76. Ioffe S, Szegedy C (2015) Batch Normalization: Accelerating Deep Network Training by Reducing Internal Covariate Shift. CoRR, vol. abs/1502.03167, [Online]. Available: https://dblp.unitrier.de/db/journals/corr/corr1502.html\#IoffeS15.

77. Srivastava N, Hinton G, Krizhevsky A, Sutskever I, Salakhutdinov R (2014) Dropout: a simple way to prevent neural networks from overfitting. J Mach Learn Res 15(56):1929-1958

78. Qin X, Yang J, Li P, Sun W, Liu W (2019) A novel relationalbased transductive transfer learning method for PolSAR images via time-series clustering. Remote Sens 11(11):1358. https://doi. org/10.3390/rs11111358

79. Chattopadhyay R, Sun Q, Fan W, Davidson I, Panchanathan S, Ye J (2012) Multisource domain adaptation and its application to early detection of fatigue. ACM Trans Knowl Discov Data 6(4):1-26. https://doi.org/10.1145/2382577.2382582

80. Ben-David S, Blitzer J, Crammer K, Kulesza A, Pereira F, Vaughan JW (2010) A theory of learning from different domains. Mach Learn 79(1-2):151-175. https://doi.org/10.1007/s10994009-5152-4

81. Zhao P, Hoi SCH, Wang J, Li B (2014) Online transfer learning. Artif Intell 216:76-102. https://doi.org/10.1016/j.artint.2014.06. 003

82. Tao J, Chung F, Wang S (2012) A kernel learning framework for domain adaptation learning. Sci China Inf Sci 55(9):1983-2007. https://doi.org/10.1007/s11432-012-4611-x

83. Pan SJ, Yang Q (2010) A survey on transfer learning. IEEE Trans Knowl Data Eng 22(10):1345-1359. https://doi.org/10.1109/ TKDE.2009.191

84. Burger A, Eyraud F (2013) "CID Image Data Portal: Architecture and Infrastructure," ec-jrc, JRC98776

85. Bishop CM (1995) Neural networks for pattern recognition. Clarendon Press, Oxford University Press, Oxford, New York

86. Goodfellow I, Bengio Y, Courville A (2016) Deep learning. The MIT Press, Cambridge, Massachusetts

87. Congalton RG (1991) A review of assessing the accuracy of classifications of remotely sensed data. Remote Sens Environ 37(1):35-46

88. Maggiori E, Tarabalka Y, Charpiat G, Alliez P (2017) Convolutional neural networks for large-scale remote-sensing image classification. IEEE Trans Geosci Remote Sens 55(2):645-657. https://doi.org/10.1109/TGRS.2016.2612821

Publisher's Note Springer Nature remains neutral with regard to jurisdictional claims in published maps and institutional affiliations. 